\title{
Centrifuge modelling of tunnelling induced ground displacements: pressure and displacement control tunnels
}

\author{
Geyang Song ${ }^{\mathrm{a}, *}$, Alec M. Marshall ${ }^{\mathrm{a}}$ \\ ${ }^{a}$ Faculty of Engineering, University of Nottingham, University Park, NG7 2RD \\ Nottingham, United Kingdom.
}

\begin{abstract}
Predicting the impact of tunnelling-induced ground movements on existing foundation systems or infrastructure is an important stage during tunnel design or risk assessment processes. Centrifuge modelling has been used extensively as a tool to study soil movements caused by tunnelling and their interactions with existing structures. In 2D plane-strain centrifuge models, tunnel volume loss can be simulated in a variety of ways, but is conventionally done using a fluid-filled flexible membrane or a rigid boundary mechanical model tunnel. The choice of model tunnel has an impact on the imparted tunnel boundary displacements and resulting ground deformations, yet a thorough quantitative evaluation of these effects has not been conducted. This paper aims to address this by contrasting plane-strain centrifuge test results from experiments using a flexible membrane model tunnel with those from a newly developed eccentric rigid boundary mechanical model tunnel. A quantitative
\end{abstract}

\footnotetext{
${ }^{*}$ Corresponding author

Email addresses: geyang.song1@nottingham.ac.uk (Geyang Song), alec.marshall@nottingham.ac.uk (Alec M. Marshall)
} 
assessment of surface and subsurface settlement trough characteristics as well as soil shear and volumetric response is provided. Results from numerical analyses using a hypoplastic constitutive model are also included, focusing on evaluating centrifuge spin-up effects and contrasting numerical outcomes with experimental data relating to settlement trough characteristics and soil shear/volumetric response. The outcomes of this paper should benefit future researchers considering which type of model tunnel to adopt when developing centrifuge tests related to 2D plane-strain tunnel modelling.

Keywords: Tunnelling, Centrifuge modelling, Greenfield settlements, Numerical modelling, Hypoplastic 


\section{Highlights}

2

3

4

5

6

- Tunnelling induced ground movements for pressure and displacement control tunnels.

- Centrifuge test results of greenfield tunnelling in sands.

- Calibration of hypoplastic constitutive model for numerical analysis.

- Soil volumetric and shear strain distribution due to tunnel volume loss. 


\section{Introduction}

Tunnel construction in urban areas often occurs close to existing foundation systems or infrastructure, such as pile groups, retaining structures, and pipelines. Understanding the impact of tunnelling-induced ground movements on these critical systems is an important stage of the tunnel design or risk assessment processes.

Centrifuge modelling has been used extensively as a tool to study soil movements caused by tunnelling because of its ability to reproduce fullscale soil stress profiles within reduced scale models. Evaluating the effect of tunnelling-induced ground movements on structures is undoubtedly a three-dimensional (3D) problem. Detailed simulation of the 3D interactions between tunnel construction and existing structures can be achieved in a centrifuge, for example the miniature boring machine from Nomoto et al. (1999), or the staged de-pressurisation systems developed by Ng et al. (2013) and Gue and Elshafie (2019). These methods add a level of complexity to the modelling process and, in the case of the mechanical excavation systems, require a significant amount of space within the centrifuge cradle. As a result, centrifuge models of the tunnelling process often simplify the problem by considering the two-dimensional (2D) displacements (horizontal and vertical) in the direction transverse to the longitudinal axis of the tunnel (i.e. the plane-strain condition representing displacements a certain distance behind the excavation face). Whilst this approach has limitations compared to the real 3D scenario (Franza et al., 2019b), 2D centrifuge modelling has provided valuable data to improve understanding of soil and soil-structure interaction behaviour for tunnelling problems. 
One of the early 2D plane-strain techniques used to simulate the effect of tunnel volume loss in centrifuge tests involved decreasing the internal pressure of a tunnel-shaped airbag (Atkinson et al., 1975; Potts, 1976; Hagiwara et al., 1999). A limitation of the pressurised airbag technique is that the compressibility of air makes it difficult to assess the magnitude of tunnel volume loss $V_{l, t}$ (i.e. the ground loss at the boundary of the tunnel). This limitation can be eliminated by replacing the air with water or oil (which may be assumed incompressible within the range of applied pressures). A concentric fluid-filled flexible membrane (FM) model tunnel has been used by several researchers (Loganathan et al., 2000; Vorster et al., 2005; Jacobsz, 2003); the concentric system implies the flexible membrane is sealed onto a shaft located centrally within the membrane. For fluid-filled FM model tunnels, the deformed tunnel shape is somewhat unknown. As discussed in Marshall and Franza (2017) and Ritter et al. (2017), the shape of the tunnel lining is controlled by the equilibrium condition between the pressures from the soil and the fluid within the tunnel. Displacements around real shallow tunnels have been shown to take an eccentric shape, with greater displacements occurring at the tunnel crown compared to the base (invert) (Loganathan and Poulos, 1998). In order to encourage a more realistic profile of displacements, Marshall (2009); Marshall et al. (2012) modified the flexible membrane model tunnel to create an eccentric shape, where soil displacements at the invert of the tunnel were limited. The eccentric model tunnel system has been applied in several subsequent centrifuge studies related to tunnelling (Farrell, 2010; Williamson, 2014; Zhou, 2015; Franza, 2016; Ritter et al., 2017).

Another option for simulating 2D tunnel volume loss in the transverse 
plane involves the use of a rigid boundary mechanical (RBM) model tunnel. For RBM model tunnels, the deformed tunnel shape, and by implication the soil displacements around the tunnel, are known. An RBM model tunnel was developed by Katoh et al. (1998) and has been used by several researchers to study soil-structure interaction problems (Boonsiri and Takemura, 2015b,a). This mechanical tunnel imposes concentric, uniform displacements around its circumference. Shahin et al. (2011) also developed a mechanical tunnelling device with 12 segments that move vertically downwards and converge towards the tunnel centre, achieving a fixed tunnel invert during tunnel volume loss, however this device was not used within a geotechnical centrifuge. To better replicate (in a centrifuge model) the real displacements that occur around shallow tunnels (according to Loganathan and Poulos (1998)), Song et al. (2018) developed a novel eccentric rigid boundary mechanical (eRBM) model tunnel that is able to induce an eccentric profile of soil displacements around the tunnel, predominately near the tunnel crown (results from tests using this model tunnel are presented in this paper).

Marshall and Franza (2017) showed, based on the data from Boonsiri and Takemura (2015b), that the set of equations provided by Marshall et al. (2012) for the prediction of settlement width parameters does not always provide a good fit to data obtained using a concentric RBM model tunnel. It was difficult to isolate a specific reason for the differences, since the variation of such factors as soil type, sample preparation method, and measurement errors between the different studies inevitably affected results. However, Marshall and Franza (2017) suggested that the different tunnel boundary conditions between the data sets (FM in Marshall (2009); Franza (2016); 
Zhou et al. (2014) versus displacement control RBM lining in Boonsiri and Takemura (2015b)) was probably the most influential parameter.

It is important to have an understanding of the implications of the choice of model tunnel adopted for these types of centrifuge tests, however a thorough evaluation of this has never been conducted. This paper aims to address this shortcoming by investigating the effect of the type of centrifuge model tunnel (i.e. flexible membrane FM or rigid boundary mechanical RBM) used to simulate 2D tunnelling displacements. The paper provides details of the newly developed eccentric rigid boundary mechanical eRBM model tunnel (first presented in Song et al. (2018)) as well as data obtained using the novel eRBM model tunnel for greenfield tunnelling scenarios. Experimental results are used to explore the effect of the different tunnel boundary conditions on resulting ground deformations and shear/volumetric response. Results from 2D finite element (FE) analyses using a hypoplastic constitutive model are also presented. The FE analyses aimed to simulate the two tunnel volume loss techniques applied in the centrifuge tests: (1) pressure controlled (replicating FM model tunnels), and (2) eccentric displacement controlled (replicating the eRBM model tunnel). Numerical results are used to explore the effect of centrifuge spin-up on outcomes from the eRBM and FM centrifuge tests, as well as evaluate the ability of the numerical model to match centrifuge test results of ground settlements and shear/volumetric response.

It is important to recognise that this paper does not attempt to make any recommendation as to which type of model tunnel best replicates reality. Each approach will have advantages and limitations, and neither gives a 
complete representation of the real tunnelling scenario. The intention of this paper is to provide quantitative evidence of the implications of the use of the different model tunnel types, providing readers with the information they need to judge which approach best suits their needs.

\section{Model tunnels}

\subsection{Flexible membrane (FM) model tunnel}

The flexible membrane model tunnel has been used extensively within centrifuge tests (Loganathan et al., 2000; Vorster et al., 2005; Jacobsz, 2003). The model tunnel used in the tests presented in this paper was a version of the eccentric flexible membrane model tunnel first used by Marshall et al. (2012) and is described in detail in Zhou (2015); Zhou et al. (2014). The model tunnel is $90 \mathrm{~mm}$ in diameter and consists of an inner aluminium core encased within a $1 \mathrm{~mm}$ thick latex membrane. Water was used to fill the annular space between the inner core and the membrane, with tunnel volume loss achieved by extracting the water using an actuator controlled by the test operator.

\subsection{Eccentric rigid boundary mechanical (eRBM) model tunnel}

In a rigid boundary mechanical (RBM) model tunnel, the displacements required to replicate tunnel volume loss are induced by controlling the displacement of the tunnel boundary. The newly developed eccentric rigid boundary mechanical eRBM model tunnel is illustrated in Figure 1. This model tunnel enables non-uniform radial displacements around the tunnel and has a maximum diameter of $90 \mathrm{~mm}$, a maximum displacement of $1.4 \mathrm{~mm}$ 


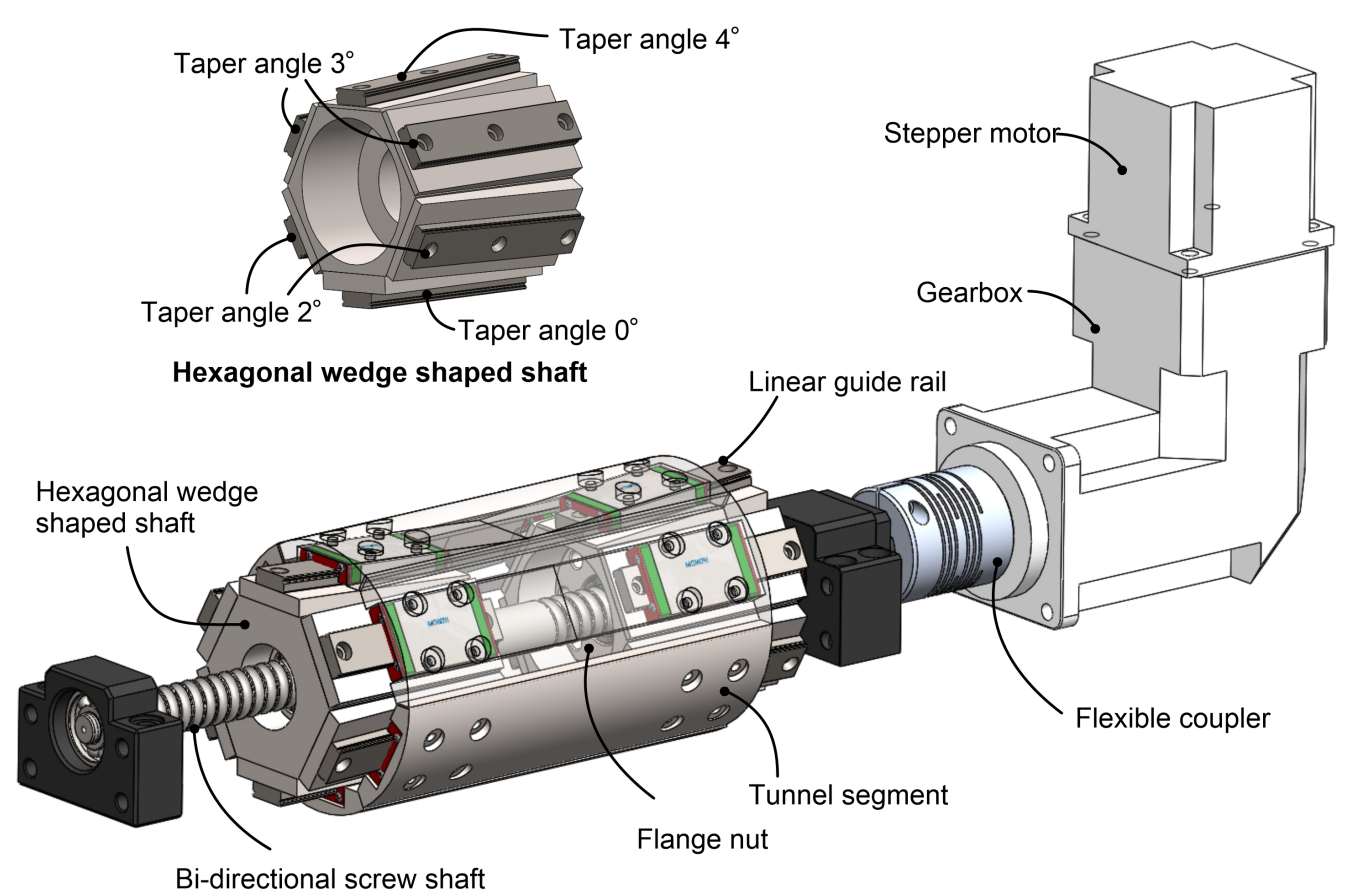

Figure 1: Newly developed eccentric rigid boundary mechanical (eRBM) model tunnel

at the tunnel crown, $0.9 \mathrm{~mm}$ at the springline, and zero displacement at the tunnel invert.

The tunnel contains a single bi-directional screw shaft which is equipped with two flange nuts. Two hexagonal wedge-shaped shafts are fixed into the bi-directional ball screw flange nuts in opposite directions. Six tunnel segments representing the tunnel boundary are connected to the hexagonal wedge-shaped shafts via six linear guide rails; these enable the relative movement between the segment and shaft along the wedge taper angle. The bi-directional screw shaft is driven by a stepper motor through a 1:10 ratio gearbox. Tunnel volume loss is achieved by rotating the bi-directional screw shaft, which causes the two hexagonal wedge-shaped shafts to move in op- 
posite directions, causing the relative movement between the six individual segments according to the different taper angles of the wedge-shaped shafts. The wedge-shaped shaft has six surfaces, where the taper angle varies from $4^{\circ}$ at the tunnel crown to $0^{\circ}$ at the tunnel invert. The six tunnel segments move eccentrically towards the tunnel centreline and create the desired non-uniform tunnel boundary displacement. Figure 2 shows the positions of the six tunnel segments at the initial and final stages of testing. The tunnel volume loss can be related to the horizontal displacement of the wedge-shaped shafts, which is measured using a linear variable differential transformer (LVDT). In tests, the computer controlled stepper motor was set to rotate by increments of $450^{\circ}$, which corresponds to $0.625 \mathrm{~mm}$ (1/8 of screw pitch) horizontal movement of the wedge-shaped shafts and $0.18 \%$ of tunnel volume loss. The maximum horizontal displacement of the wedge-shaped shafts is around $20 \mathrm{~mm}$, giving a maximum tunnel volume loss of $3.5 \%$.

A gap is created between adjacent segments that make up the tunnel circumference, as illustrated in Figure 2, creating a 'step' between segments during tunnel volume loss. The maximum size of the 'step' (at maximum tunnel volume loss) is $0.3 \mathrm{~mm}$. The gaps are initially $2 \mathrm{~mm}$ (before tunnel volume loss) and vary with tunnel volume loss at different rates for the various gaps. Copper strips were placed along the gaps in the direction of the tunnel length to create a smooth tunnel surface, as shown in Figure 2. The tunnel was then sealed within two latex membranes to protect it from soil intrusion during tests; this also ensured a smooth variation of soil deformation was achieved across the gaps. 


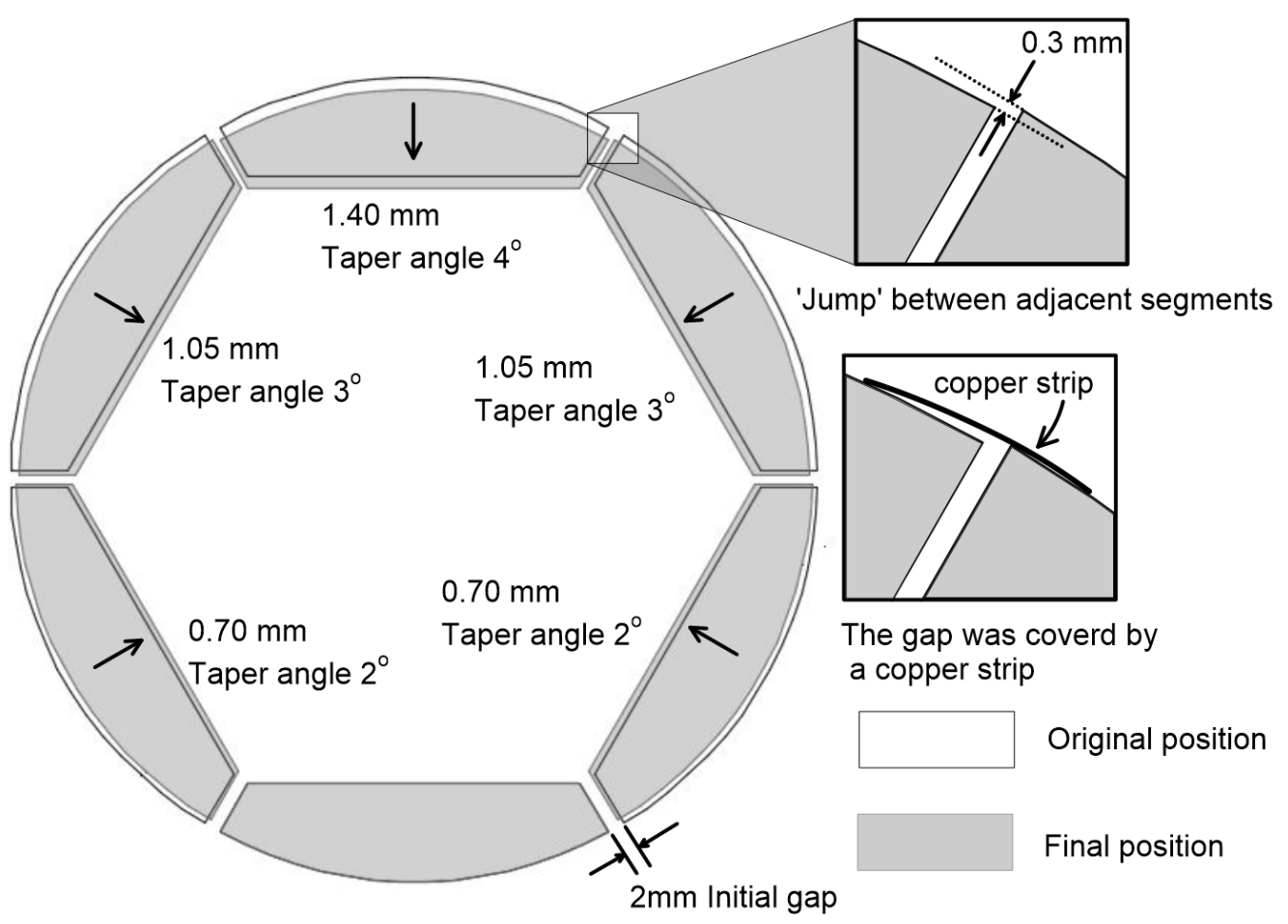

Figure 2: eRBM model tunnel deformed shape 


\section{Centrifuge modelling}

This paper uses data from six centrifuge tests, as described in Table 1. The flexible membrane (FM) test data were obtained from previous studies (Xu et al., 2019; Zhou, 2015; Marshall, 2009), whereas the eRBM centrifuge test data were newly acquired as part of the current study. A fine-grained silica sand commonly known as Leighton Buzzard Fraction E sand was used for all tests, which has a typical average diameter $D_{50}$ of $0.14 \mathrm{~mm}$ and a specific gravity $G_{s}$ of 2.65 . The sand has a maximum $\left(e_{\max }\right)$ and minimum $\left(e_{\min }\right)$ void ratio of 1.01 and 0.61 , respectively, and a coefficient of uniformity $C_{u}$ of 1.58 (Tan, 1990). Details of the preparation methodologies for the FM tests can be found in the references provided in Table 1. This section is mainly dedicated to describing the new eRBM centrifuge tests. In this paper, dimensions of the models and results are described in model scale, unless otherwise stated.

Note that the tests presented in Table 1 are not all consistent in terms of tunnel size and centrifuge scaling factor. As such, soil displacements for a given $C / D_{t}$ ratio at a given tunnel volume loss will not be wholly consistent (i.e. for the same magnitude of tunnel volume loss, a larger tunnel will incur larger soil displacements than a smaller tunnel) and the levels of soil dilation around deeper tunnels would be less than for shallower tunnels, which would affect the characteristics of the settlement troughs above the tunnel. The implications of this will be discussed in detail later in the paper.

The three eRBM centrifuge tests were carried out on the University of Nottingham Centre for Geomechanics (NCG) 2 m radius, 50 g-tonne geotechnical centrifuge at an acceleration of $80 \mathrm{~g}$. The model strongbox has inner 
Table 1: Centrifuge test details in model scale [prototype scale in parentheses]

\begin{tabular}{lcccc}
\hline Test name & $C(\mathrm{~mm})[\mathrm{m}]$ & $D_{t}(\mathrm{~mm})[\mathrm{m}]$ & $\sigma_{v, t}(\mathrm{kPa})[\mathrm{same}]$ & Reference \\
\hline FM $C / D_{t}=1.3$ & $120[8.160]$ & $90[6.12]$ & 172 & Xu et al. (2019) \\
FM $C / D_{t}=2.0$ & $180[14.400]$ & $90[7.2]$ & 275 & Zhou (2015) \\
FM $C / D_{t}=2.4$ & $151[11.325]$ & $62[4.65]$ & 209 & Marshall (2009) \\
eRBM $C / D_{t}=1.3$ & $117[9.360]$ & $90[7.2]$ & 198 & this study \\
eRBM $C / D_{t}=2.0$ & $180[14.400]$ & $90[7.2]$ & 275 & this study \\
eRBM $C / D_{t}=2.4$ & $216[17.280]$ & $90[7.2]$ & 319 & this study \\
\hline
\end{tabular}

$C=$ depth to tunnel crown; $D_{t}=$ tunnel diameter; $\sigma_{v, t}=$ vertical stress at tunnel axis depth.

width $\times$ height dimensions of $700 \mathrm{~mm} \times 400 \mathrm{~mm}($ Figure 3) and is $150 \mathrm{~mm}$ wide. The box has an aluminium back wall and a transparent front acrylic wall that enables the acquisition of digital images of the soil during tests (used to measure sub-surface soil displacements using image analysis techniques). The model tunnel has a diameter of $90 \mathrm{~mm}$ and is located $75 \mathrm{~mm}$ above the strongbox base, giving a clear distance of $30 \mathrm{~mm}$ between the bottom of the tunnel and the strongbox base. The tunnel is fixed within the front and back walls of the centrifuge strongbox, preventing any movement of the tunnel ends during tests.

\subsection{Sample preparation}

Samples were created using the dry sand pouring technique. The tunnel was secured within the strongbox before sand pouring. To achieve a uniform sample around the model tunnel, the strong box was placed with the back wall facing downwards, allowing the sand to be poured in the direction of the tunnel longitudinal axis, with a temporary plate used to support the sand along the plane corresponding to the ground surface (consistent with 


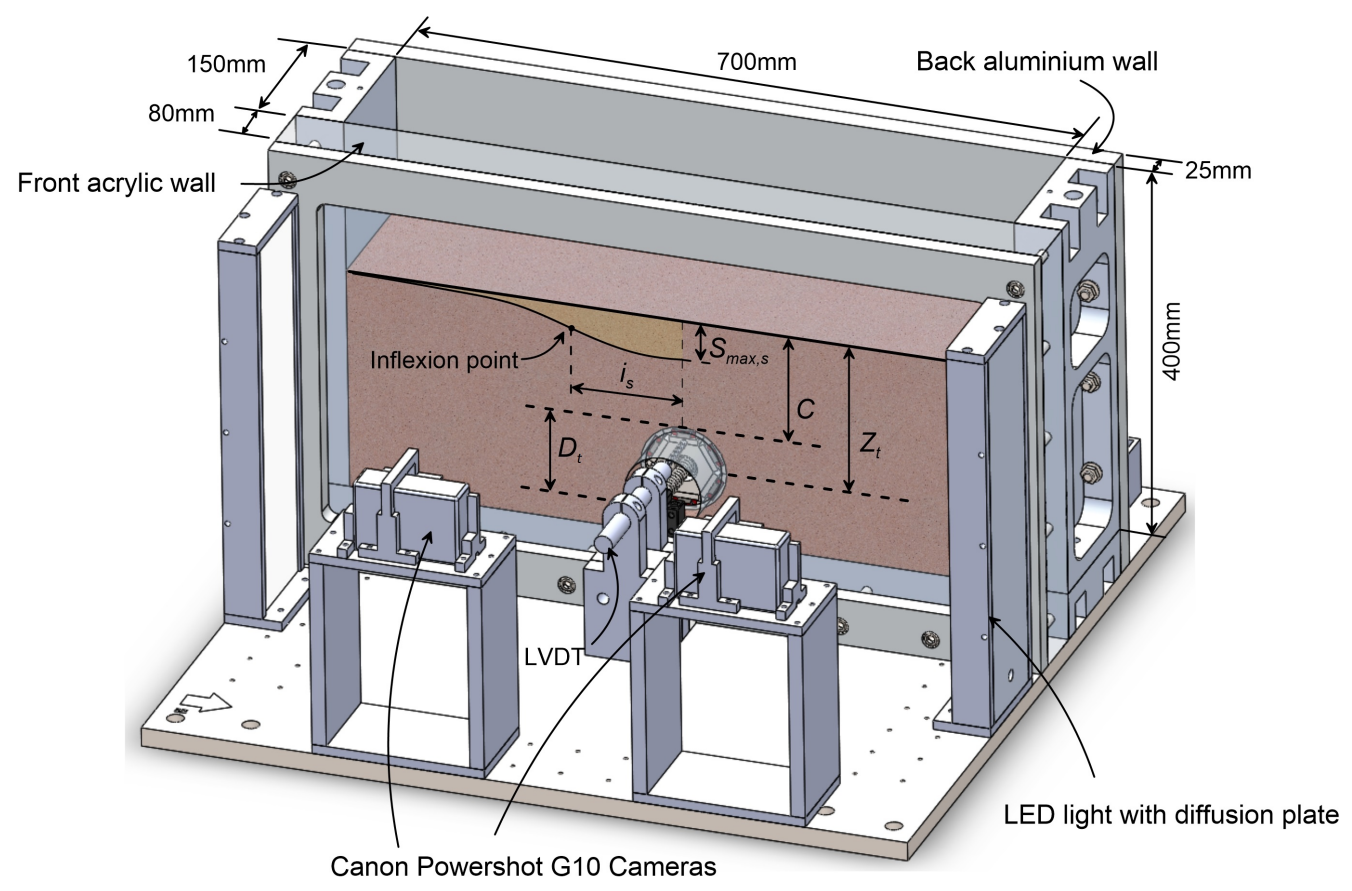

Figure 3: Centrifuge model 
Vorster (2006); Marshall (2009); Zhou (2015); Franza (2016); Farrell (2010)).

The sand was prepared according to a methodology calibrated to achieve a relative density $\left(I_{d}\right)$ of $90 \%$. After sand pouring, a thin layer of dyed sand was placed uniformly on the top surface of the sample to increase the contrast and help with the tracking of soil displacements using image analysis. The front acrylic window was then bolted to the strong box, which was then rotated to its upright position, and the temporary plate was removed, revealing the ground surface. The gearbox and stepper motor were then assembled and the LVDT placed at the front acrylic window to measure the movement of one of the wedge-shaped shafts.

\subsection{Instrumentation}

Two Canon Powershot G10 14.7 mega-pixel cameras were used to take pictures of the soil behind the acrylic wall during tests. Two LED light strips equipped with a diffusion plate were placed on either side of the strong box, providing a uniform illumination to the acrylic wall. Surface and subsurface soil movements were obtained using GeoPIV-RG (Stanier et al., 2015).

To assess the precision of the geoPIV-RG analysis based on the current set up, two images were taken at elevated gravity with zero soil displacements. For the analysis, 11844 subset patches were used, and the displacement (in both horizontal and vertical directions) of each patch was calculated. The horizontal displacement data provided a standard deviation of $4.6 \mu \mathrm{m}$, and vertical displacements provided a slightly higher standard deviation of $6.4 \mu \mathrm{m}$. This level of precision is similar to that reported by Marshall and Mair (2011) for a similar assessment procedure.

The interface friction between the sand and acrylic window can affect 
displacement measurements obtained by image analysis. The reliability of image-based soil deformation measurements in similar centrifuge tests has been studied by several researchers (Marshall et al., 2012; Elshafie et al., 2013; Ritter et al., 2017). Marshall et al. (2012) compared geoPIV displacements with LVDT measurements made within the middle of the box and demonstrated that the boundary friction did not have a significant effect on settlement trough shape. Therefore, the soil displacement data presented in this paper is based solely on image analysis measurements made at the soil-acrylic wall interface. It should also be mentioned that the comparisons of experimental data presented in this paper are all based on image analysis measurements obtained at an acrylic centrifuge container wall, hence any effects related to interface friction should be reasonably consistent across the data sets.

\subsection{Testing procedure}

The centrifuge package was spun to $80 \mathrm{~g}$ in stages of $10 \mathrm{~g}$. Three stabilisation cycles were then performed for each test, whereby the model was spun from $80 \mathrm{~g}$ to $10 \mathrm{~g}$ and then back up to $80 \mathrm{~g}$. By measuring the displacement at the ground surface during the stabilisation cycles, it was found that, for the type of soil used in these tests, three cycles was sufficient to obtain a consistent measurement of surface displacement between successive $10 \mathrm{~g}-80 \mathrm{~g}$ cycles. The stabilisation cycles help to achieve better consistency between tests by reducing localised, more highly-stressed zones ('hung-up' particles), thereby achieving a more uniformly stressed soil. After three stabilisation cycles, the tunnel volume loss process was started. Images were taken at key intervals throughout the tests (during centrifuge spin-up and tunnel volume 
loss).

\section{Numerical analyses}

To obtain a better understanding of the relationship between soil movements and the tunnel boundary condition (i.e. displacement or pressure controlled), a series of two-dimensional numerical analyses was performed to simulate eccentric displacement control (EDC) and flexible membrane (FM) model tunnels. The ABAQUS (Hibbitt, 2002) finite element analysis (FEA) software was used, along with the implementation of the hypoplastic constitutive model developed by von Wolffersdorff (1996).

\subsection{Finite element mesh and boundary conditions}

Figure 4 shows the FE mesh for tests with a cover to diameter ratio $C / D_{t}=2$, which consists of 2040 elements and 6309 nodes. Taking advantage of symmetry, only half of the model was simulated, using vertical roller boundaries on the plane of symmetry through the tunnel centreline. Potts and Zdravković (1999) indicated that for curved FE boundaries (i.e. the tunnel boundary), higher order elements should be used, hence eight-node quadratic plane strain elements (CPE8) were applied to the models. A fixed boundary was used along the bottom of the mesh, a vertical roller boundary was used on the right-side boundary (see Figure 4), and no constraints were applied to the ground surface. The dimensions of the numerical model were chosen to reduce boundary effects on the predicted displacements, hence they do not match exactly with the dimensions within the centrifuge tests (which are constrained by the size of the centrifuge cradle). It was also verified that the effect of element size on displacement measurements was negligible. 


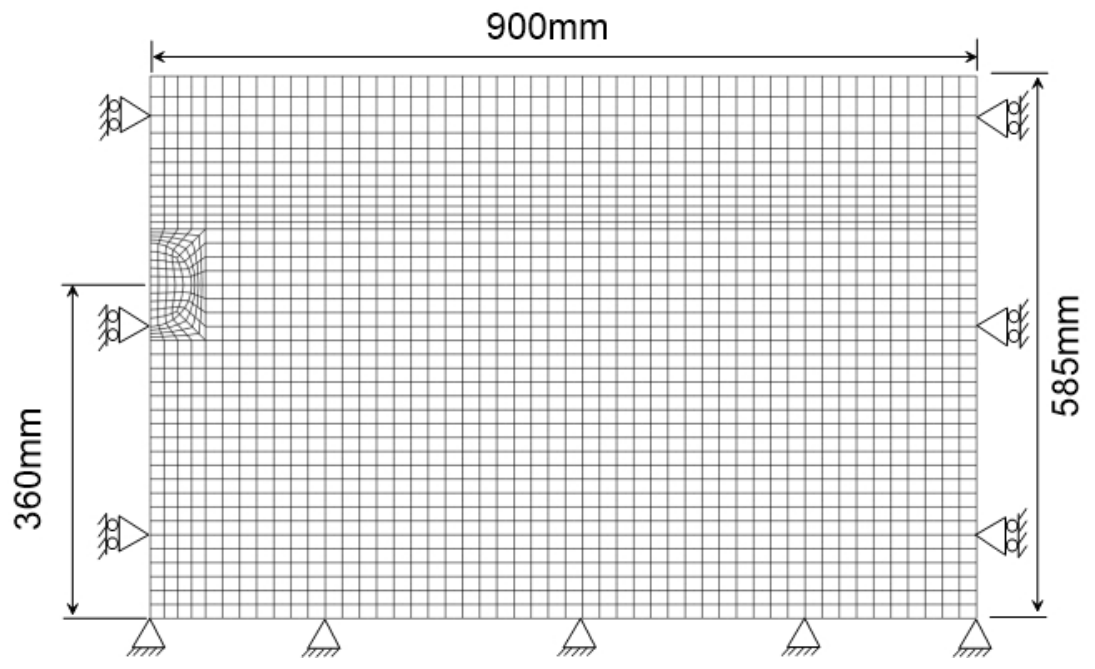

Figure 4: Mesh of the numerical (FEA) analysis for $C / D_{t}=2$

\subsection{Numerical modelling details}

The basic hypoplastic model was adopted for this study, which requires the following 8 parameters: critical state friction angle $\phi_{c}^{\prime}$, granular hardness $h_{s}$, fitting parameter $n$, minimum/maximum/critical void ratio at zero pressure $e_{d 0} / e_{i 0} / e_{c 0}$, and $\alpha ; \beta$ which govern the stiffness of the soil. The hypoplastic model parameters for Leighton Buzzard Fraction E sand were obtained and calibrated using oedometer and triaxial test data, as follows.

A critical state friction angle $\phi_{c}^{\prime}=31.4^{\circ}$ was used for the sand, based on an average value from five heap tests. Herle and Gudehus (1999) suggested that the initial void ratio in a proportional compression test (i.e. an oedometer test) with very loose sand can be considered as an appropriate estimate of $e_{c 0}$ since the sand would be poured in a similar way as a heap test, where the sample is close to critical state because of the large deformations. As shown by Herle and Gudehus (1999), $h_{s}$ can be defined using: 


$$
h_{s}=3 p^{\prime}\left(\frac{n e_{p}}{C_{c}}\right)^{1 / n}
$$

where $C_{c}$ is the tangent compression index $\left(C_{c}=\Delta e / \Delta \ln p^{\prime}\right)$.

Using data from an oedometer test and considering two values of $C_{c}$ at different magnitudes of mean effective stress $p^{\prime}$ (i.e. two points on an $e-\ln \left(p^{\prime}\right)$ curve; indicated below by subscripts 1 and 2), Equation 1 can be used to obtain an expression for $n$ :

$$
n=\frac{\ln \left(e_{1} C_{c 2} / e_{2} C_{c 1}\right)}{\ln \left(p_{2}^{\prime} / p_{1}^{\prime}\right)}
$$

Using the above approach on data obtained from an oedometer test on Fraction E sand (see Figure 5), values of $e_{c 0}=1.16, h_{s}=1969 \mathrm{MPa}$ and $n=0.447$ were obtained.

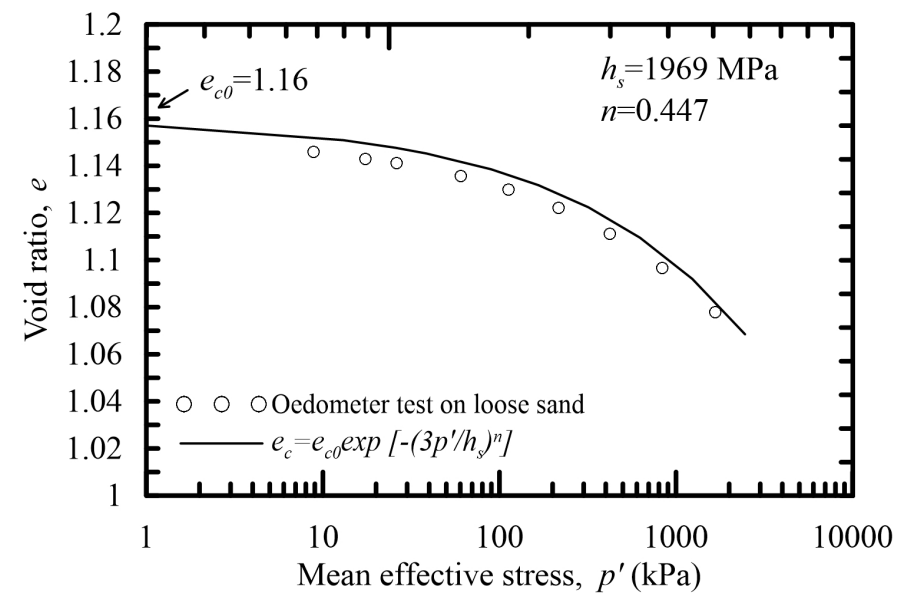

Figure 5: Oedometer test data for Leighton Buzzard Fraction E sand

The value of minimum void ratio at zero pressure $e_{d 0}$ can be determined 
using (Herle and Gudehus, 1999):

$$
e_{d 0}=e_{d} \exp \left[\left(3 p^{\prime} / h_{s}\right)^{n}\right]
$$

where the value of $e_{d}$ mainly depends on the coefficient of uniformity $C_{u}$ and grain shape. Youd (1973) measured the value of $e_{d}$ using a simple shear test under a vertical pressure of $96 \mathrm{kPa}$, corresponding to $p^{\prime} \approx 55 \mathrm{kPa}$ (based on assumption of $K=0.4$ ), and proposed a diagram relating $e_{d}$ to grain angularity and $C_{u}$. Based on the diagram of Youd (1973), a value of $e_{d}=$ 0.615 was obtained. By substituting this value into Equation 3 (along with previously determined parameter values), a value of $e_{d 0}=0.624$ was obtained. As suggested by Herle and Gudehus (1999), the value of maximum void ratio at zero pressure $e_{i 0}$ can be approximated as $e_{i 0} \approx e_{c 0} \times 1.2$, therefore, the critical void ratio at zero pressure $e_{i 0}$ was determined as 1.392 .

Two drained triaxial compression tests were conducted at an effective confining pressure of $200 \mathrm{kPa}$ using the same sand that was used in the centrifuge tests, with the same relative density of $I_{d}=90 \%$. To calibrate the parameters $\alpha$ and $\beta$, a single 3D cuboid element (C3D8) numerical analysis was conducted in ABAQUS to replicate the triaxial tests. Figure 6 shows that numerical simulation results using $\alpha=0.065$ and $\beta=0.3$ compare well against both the triaxial volumetric data and the non-linear stress-strain response of the soil. Table 2 summarises the Leighton Buzzard Fraction E sand parameters adopted in this study for the basic hypoplastic model.

The intention of the FE analyses was to simulate, as closely as possible, the conditions within the various centrifuge tests, considering: (1) the effect of increasing acceleration within the models from $1 \mathrm{~g}$ to $80 \mathrm{~g}$, and (2) the tunnel volume loss process. In relation to (1), two modelling approaches 

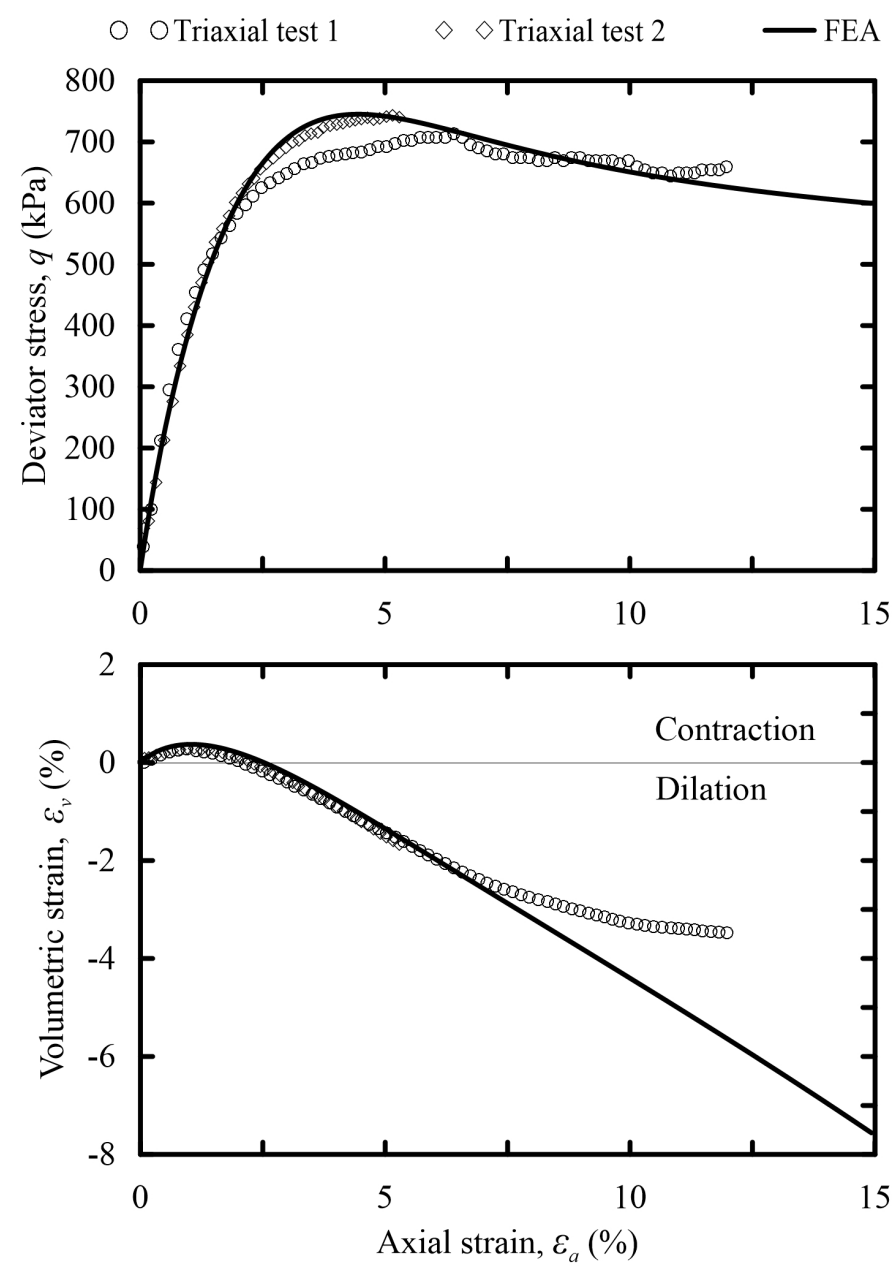

Figure 6: Drained triaxial test data for Leighton Buzzard Fraction E sand and numerical (FEA) results using hypoplastic model (effective confining stress $=200 \mathrm{kPa}$ ) 
Table 2: Adopted hypoplastic model parameters for Leighton Buzzard Fraction E sand

\begin{tabular}{lcc}
\hline Parameter & Value & Source \\
\hline Critical state friction angle $\phi_{c}^{\prime}$ & $31.4^{\circ}$ & Heap test \\
Granular hardness $h_{s}$ & $1969 \mathrm{MPa}$ & Oedometer test \\
Exponent $n$ & 0.447 & Oedometer test \\
Minimum void ratio at zero pressure $e_{d 0}$ & 0.624 & Herle and Gudehus (1999) \\
Critical void ratio at zero pressure $e_{c 0}$ & 1.16 & Oedometer test \\
Maximum void ratio at zero pressure $e_{i 0}$ & 1.392 & Herle and Gudehus (1999) \\
Exponent $\alpha$ & 0.065 & Triaxial test \\
Exponent $\beta$ & 0.3 & Triaxial test \\
\hline
\end{tabular}

were adopted: first, where the full $1 \mathrm{~g}$ to $80 \mathrm{~g}$ process was replicated, and second, where the $80 \mathrm{~g}$ stress conditions were imposed on the model as an initial condition (referred to as ' $80 \mathrm{~g}$ initially'). It was found that, when replicating the full $1 \mathrm{~g}$ to $80 \mathrm{~g}$ process, the numerical analyses were unstable (i.e. numerical convergence issues) for tunnel volume losses greater than $V_{l, t}=1.8 \%$. By replicating from $2 \mathrm{~g}$ to $80 \mathrm{~g}$, numerical analyses could be achieved up to $V_{l, t}=2 \%$, hence results from models which replicated the stress increase from $2 \mathrm{~g}$ to $80 \mathrm{~g}$ are included in this paper (referred to as ' $2-80 \mathrm{~g}$ considered'). Comparison of results between '1-80 g' and '2-80 g' analyses at $V_{l, t}=1.8 \%$ showed that the difference in ground settlements were negligible. In relation to (2), two tunnel volume loss simulation methodologies were undertaken: the eccentric displacement controlled (EDC) method (Cheng et al., 2007) to simulate the eccentric rigid boundary mechanical (eRBM) model tunnel, wherein the tunnel invert was fixed against translation to replicate the conditions of the eRBM centrifuge tests, and the pressure controlled 
method (PCM) to simulate flexible membrane (FM) model tunnels.

The FE analysis simulation procedures for the ' $2-80 \mathrm{~g}$ considered' models can be summarised as follows. A geostatic step was conducted to impose a predefined stress profile $\left(K_{0}=0.5\right)$ within the soil elements under $2 \mathrm{~g}$, after which soil displacements were reset to zero (the maximum soil displacement after the geostatic step was found to be $2 \times 10^{-5} \mathrm{~mm}$ ). The soil elements inside the tunnel were then deactivated. For the EDC numerical analyses, a tunnel boundary was used with a frictionless interface with the soil. Gravity was then increased from $2 \mathrm{~g}$ to $80 \mathrm{~g}$, where the rigid boundary of the model tunnel was fixed in terms of both distortion (change in shape) and translation (rigid body motion). For the PCM analyses, the internal tunnel pressure was increased according to the g-level, accounting as well for the gradient of stress due to the self-weight of water within the model tunnel. Upon reaching $80 \mathrm{~g}$, the simulation of tunnel volume loss was then conducted. For the EDC analyses, a non-uniform displacement profile was imposed on the rigid boundary elements by assuming zero displacement at the tunnel invert and maximum displacements at the crown, with displacements oriented towards the tunnel centre (identical to the eRBM model tunnel centrifuge tests; see deformed tunnel shape in Figure 2). For PCM numerical analyses, the tunnel volume loss was replicated by gradually decreasing the internal tunnel pressure.

For the ' $80 \mathrm{~g}$ initially' numerical analyses, the geostatic step was conducted for stress conditions corresponding to $80 \mathrm{~g}$ and $K_{0}=0.5$; all other details were the same as the '2-80 g considered' analyses. 


\section{Results}

\subsection{Effect of model tunnel on initial conditions}

Before considering tunnel volume loss in detail, it is of interest to investigate the effect of the increased self-weight in the centrifuge on the displacements and stress state of the soil around the model tunnels (i.e. the effect of centrifuge spin-up). For FM model tunnel experiments (centrifuge tests), the internal tunnel pressure was set to an approximation of the vertical overburden pressure at the tunnel axis depth using a stand-pipe system. The difference in self-weight and lateral earth pressure between the soil and water $\left(K_{0}=1\right.$ for water; $K_{0}$ assumed to be 0.5 for soil) will cause an imbalance of stresses in the region around the model tunnel (also discussed by Ritter et al. (2017)). PCM numerical analyses were conducted to investigate soil deformations and ground stress changes as the soil self-weight was increased during centrifuge spin-up from $2 \mathrm{~g}$ to $80 \mathrm{~g}$. For the eRBM model tunnel, the tunnel lining was fixed in place (displacement controlled), hence limited displacement is expected for the soil close to the tunnel lining during centrifuge spin-up.

Figure 7 shows the centrifuge and numerical (FEA) data for soil movements around the tunnels during centrifuge spin-up. The vertical displacements in Figure 7(a) (positive displacement is downwards) show that the soil moves downwards in the region above the tunnel crown for both FM and eRBM centrifuge tests. For the EDC (eccentric displacement controlled) FE analyses, since the tunnel boundary is fixed in place, the soil settlements around the tunnel periphery are less than for the PCM numerical analyses (note the different contour scale for this plot). 
From the horizontal displacement data in Figure 7(b), despite the difference in g-level increase (i.e. $1-80 \mathrm{~g}$ for centrifuge and $2-80 \mathrm{~g}$ for numerical FEA), both centrifuge and numerical (FEA) results for the FM/PCM tunnels show outwards soil movements close to the tunnel springline (negative displacement is to the left). This happens because the internal tunnel pressure at the tunnel springline is greater than the horizontal soil pressure, causing the tunnel boundary to push outwards at the springline until an increased passive earth pressure is achieved to balance the internal tunnel pressure. The numerical PCM results show greater horizontal soil movements than the FM centrifuge test (note the larger contour scale used for FEA PCM in Figure $7(\mathrm{~b})$ ), suggesting that the assumed $K_{0}$ for the soil may have been underestimated ( $K_{0}$ assumed to be 0.5 for soil, consistent with Marshall et al. (2010); Haji et al. (2018)). For the eRBM centrifuge model tunnel, the overall shape does not change during centrifuge spin-up, but outwards movements of the soil in the region of the tunnel shoulders are observed from the FEA EDC results. The centrifuge horizontal displacements for the eRBM do not show a clear mechanism with increase in g-level.

To gain a better understanding of what happens within the soil around flexible membrane (FM) and eccentric rigid boundary mechanical (eRBM) model tunnels during gravity increase, as well as during the subsequent tunnel volume loss process, Figure 8 plots the numerical (FEA) results of horizontal and vertical stress paths of soil elements near the tunnel crown and springline for pressure controlled (PCM) and eccentric displacement controlled (EDC) simulations with $C / D_{t}=1.3$ (positive stresses are compressive). The two modelling approaches are included: (1) the ' $2-80 \mathrm{~g}$ considered' model and (2) 

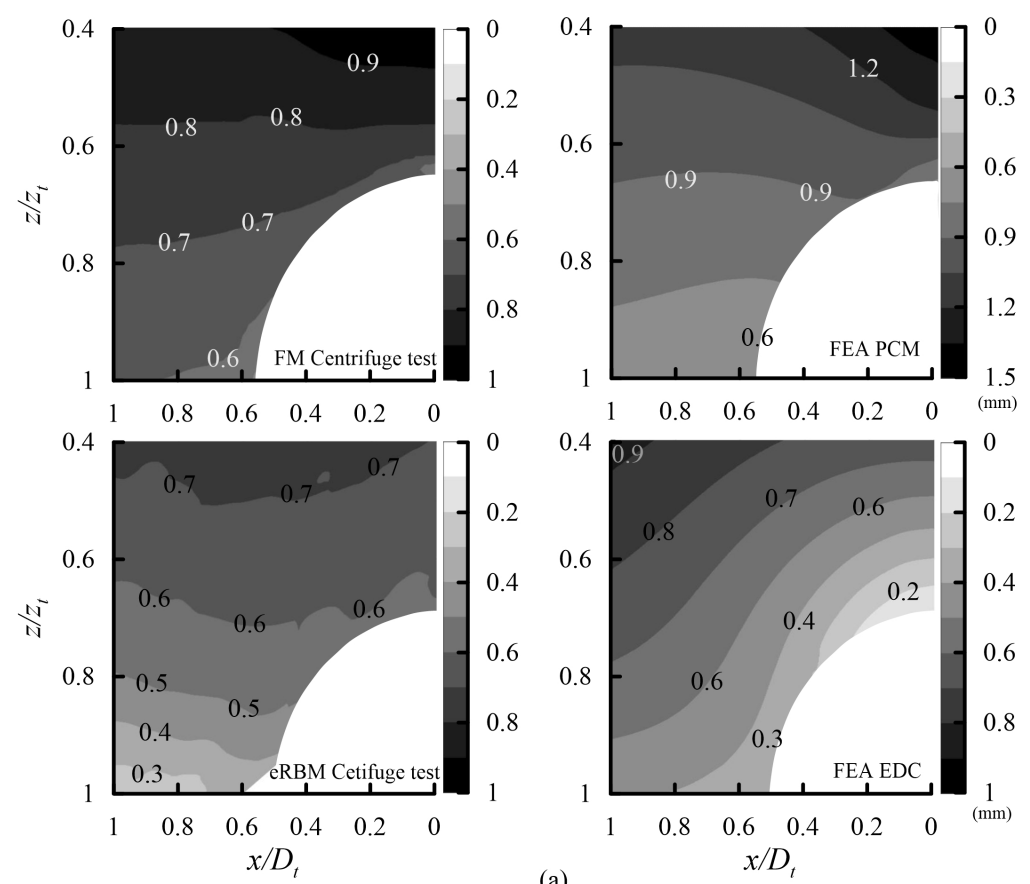

(a)
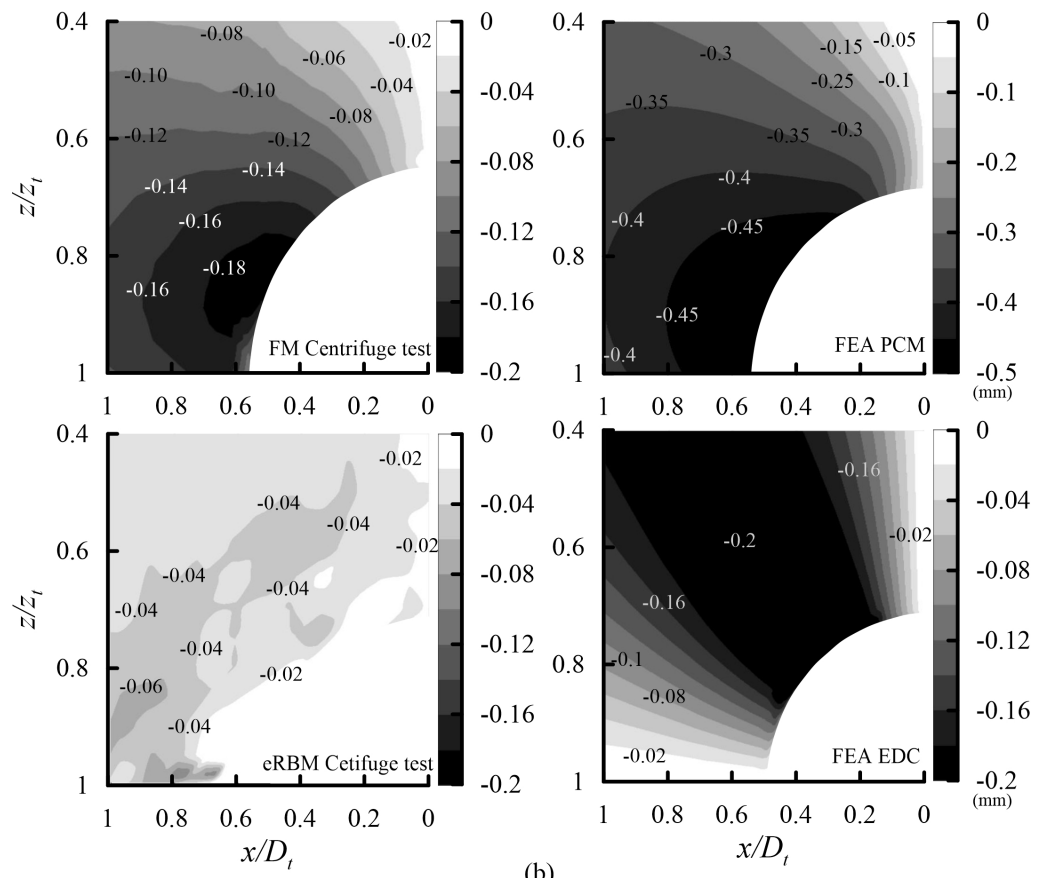

(b)

Figure 7: Soil movements around FM and eRBM model tunnels during centrifuge spin-up: (a) vertical displacements (positive downwards), (b) horizontal displacements (negative towards the left) 
the ' $80 \mathrm{~g}$ initially' model (the $K_{0}$ line with no tunnel is also shown in Figure 8). The simulation of tunnel volume loss up to $V_{l, t}=2 \%$ was then performed for both models. For the solid circles in Figure 8(a), the stress states in the '2$80 \mathrm{~g}$ considered' models vary according to the soil element locations (close to tunnel crown or springline), whereas for the solid circles in Figure 8(b) (' $80 \mathrm{~g}$ initially' models), the stress states at $80 \mathrm{~g}$ all fall along the $K_{0}$ line. Prior to tunnel volume loss in Figure 8(b), there is an intermediate stage (solid circles) in both the EDC and PCM models. For the EDC model, the intermediate stage relates to the stress adjustments caused by the introduction of the frictionless rigid tunnel boundary element. For the PCM model, the stage is related to the stress imbalance caused by the different horizontal earth pressures within the sand and the water inside the model tunnel (as discussed by Ritter et al. (2017)); this is referred to below as 'stress re-balance'. The intermediate stage is then followed by tunnel volume loss up to $2 \%$, indicated by the open circles.

Considering the ' $80 \mathrm{~g}$ initially' results in Figure $8(\mathrm{~b})$, the PCM model stress state near the springline starts on the $K_{0}$ line (grey solid line). Horizontal stress then increases considerably and the vertical stress decreases slightly during the 'stress re-balance' process. The stress state at the solid grey circle is very close to the initial state of the ' $2-80 \mathrm{~g}$ considered' model in Figure 8 (a). The stress paths of the ' $2-80 \mathrm{~g}$ considered' and the ' $80 \mathrm{~g}$ initially' models then follow near-identical paths during the tunnel volume loss process (proceeding to the open grey circles). For the stress paths of the PCM models near the crown, the vertical stress state of the '2-80 g considered' model in Figure 8 (a) is slightly lower than the ' $80 \mathrm{~g}$ initially' model (solid grey circle) 


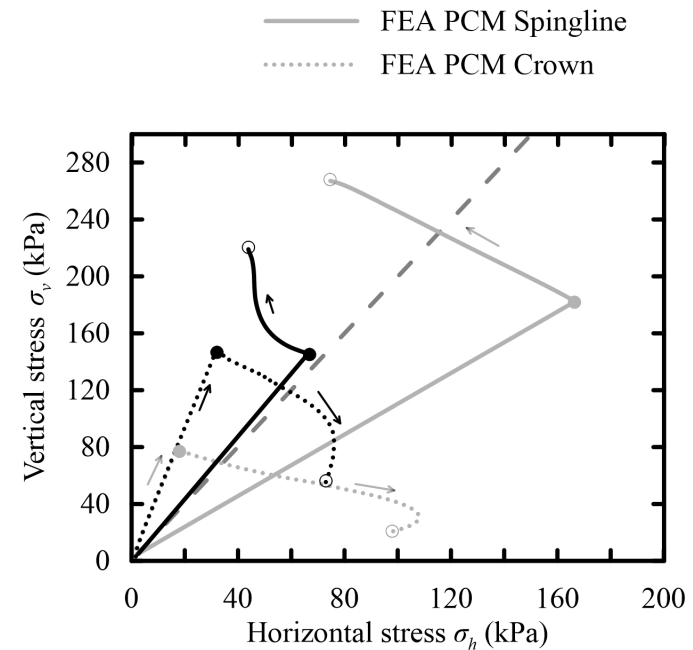

(a)

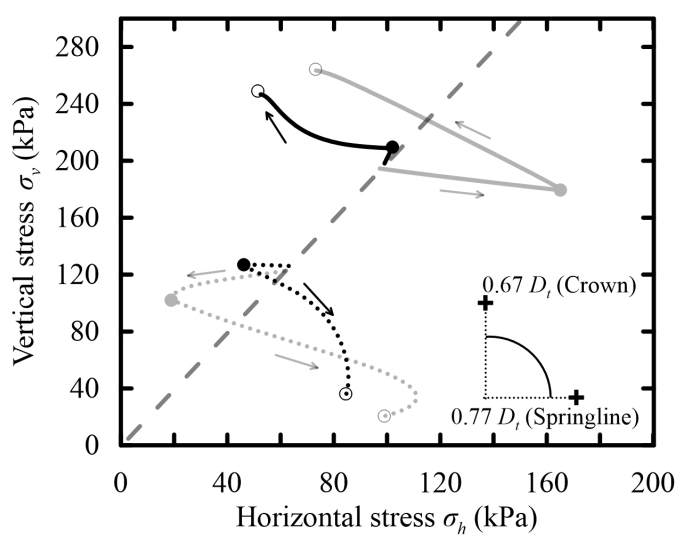

(b)

Figure 8: Vertical and horizontal stress paths for $C / D_{t}=1.3$ : (a) 2-80 g considered method, (b) $80 \mathrm{~g}$ initially method

in $8(\mathrm{~b})$; the horizontal stresses are nearly the same. The stress paths during volume loss follow a similar path and ultimately (at $2 \%$ volume loss) reach the same location (grey open circle). For the EDC models, a clear offset between the stress states prior to tunnelling in the ' $2-80 \mathrm{~g}$ considered' and ' $80 \mathrm{~g}$ initially' models can be seen, but the shape of the stress paths during tunnel volume loss is similar.

The impact of the different modelling approaches on profiles of ground settlement, $S_{v}$, at three values of normalised depth, $z / z_{t}$, is provided in Figure 9 at a tunnel volume loss of $V_{l, t} \approx 2 \%$. For the PCM model, as the value of internal tunnel pressure controls the volume loss, the magnitude of tunnel volume loss was calculated based on the displacement of the nodes along the tunnel lining.

In general, for a given type of model tunnel (i.e. EDC or PCM), the 
shape of the settlement troughs between the ' $2-80 \mathrm{~g}$ considered' and ' $80 \mathrm{~g}$ initially' models is similar. However, the magnitude of settlement for the ' $2-80 \mathrm{~g}$ considered' models is always slightly greater than the ' $80 \mathrm{~g}$ initially' models (by up to about $15 \%$ at the surface). With an increase in depth, the difference in settlement magnitude between the two modelling approaches (i.e. '2-80 g considered' versus ' $80 \mathrm{~g}$ initially') becomes smaller. Comparing results between EDC and PCM models, the PCM model provided a greater settlement than the EDC for the soil close to the tunnel centreline $\left(x / D_{t}<\right.$ $1.5)$.

To summarise: (1) the PCM model tends to generate a 'steeper' displacement profile with larger maximum settlement than the EDC model, and (2) the magnitude of settlement for the ' $2-80 \mathrm{~g}$ considered' models is always greater than the ' $80 \mathrm{~g}$ initially' models for both EDC and PCM models, however this difference tends to decrease with depth.

\subsection{Effects on soil deformation mechanisms}

For a given tunnel volume loss, the shape and magnitude of soil displacements are affected by the type (displacement/pressure controlled) and displaced shape of the tunnel lining. For eccentric displacement controlled model tunnels (EDC), the displaced tunnel shape is pre-defined, whereas for flexible membrane FM model tunnels, the tunnel boundary shape is free to deform according to the internal/external stresses applied to it. The effect of these differences on resulting ground displacements are considered in this section using both centrifuge test and numerical analysis data (based on the '2-80g considered' method presented in the previous section).

Figure 10 compares soil displacements from the eccentric rigid boundary 

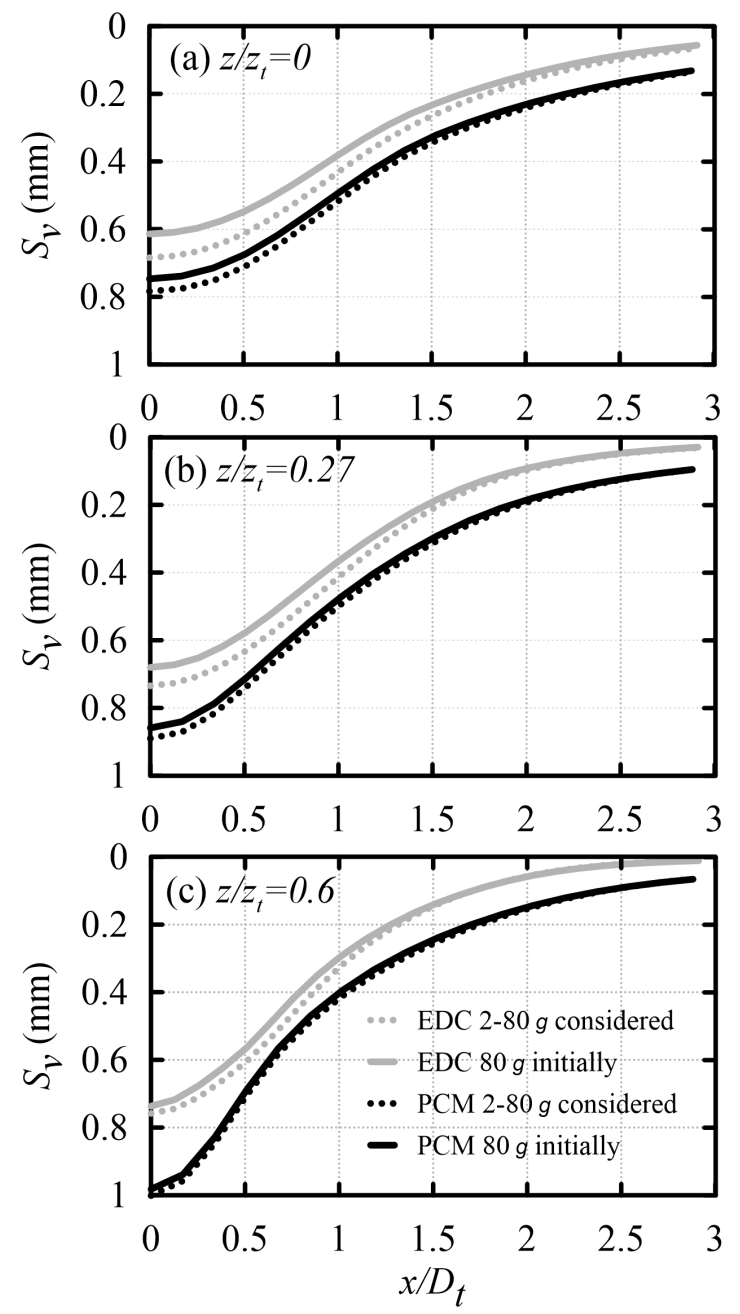

Figure 9: Settlement trough for $C / D_{t}=1.3$ at $V_{l, t} \approx 2.0 \%$ : (a) $z / z_{t}=0$, (b) $z / z_{t}=0.27$, (c) $z / z_{t}=0.6$ 
mechanical eRBM model tunnel centrifuge test with those from a flexible membrane FM centrifuge test (Franza, 2016) for $C / D_{t}=2$ at a tunnel volume loss of $V_{l, t}=2 \%$; note that these two tests are identical except for the model tunnel type (refer to Table 1). For vertical displacements, the deformation patterns match well between the tests (eRBM and FM); however, the eRBM settlement trough is slightly wider than the FM trough. For horizontal displacements, the FM model tunnel causes a zone of relatively high magnitude displacements above and slightly to the side of the tunnel crown, as well as a major zone of displacements at the ground surface with a maximum value similar to that near the tunnel. The zone of large horizontal displacements for the eRBM tunnel is located closer to the tunnel springline compared with the FM model tunnel. There is also a large zone of horizontal displacements at the ground surface for the eRBM tunnel, in a similar location but more widespread than for the FM tunnel; the magnitude of displacements at the ground surface is noted to be less than at the springline for the eRBM tunnel.

Figure 11 shows the numerical (FEA) results of vertical and horizontal soil displacements for simulations of the different model tunnels at $C / D_{t}=2$. As previously mentioned, the pressure controlled method (PCM) was used to simulate the flexible membrane (FM) model tunnel, and the eccentric displacement controlled method (EDC) was used to replicate the eccentric rigid boundary mechanical (eRBM) model tunnel.

The vertical and horizontal soil displacements in Figure 11 using FEA PCM can be compared with the FM model tunnel centrifuge test data in Figure 10. The general pattern of vertical displacements from the FEA 

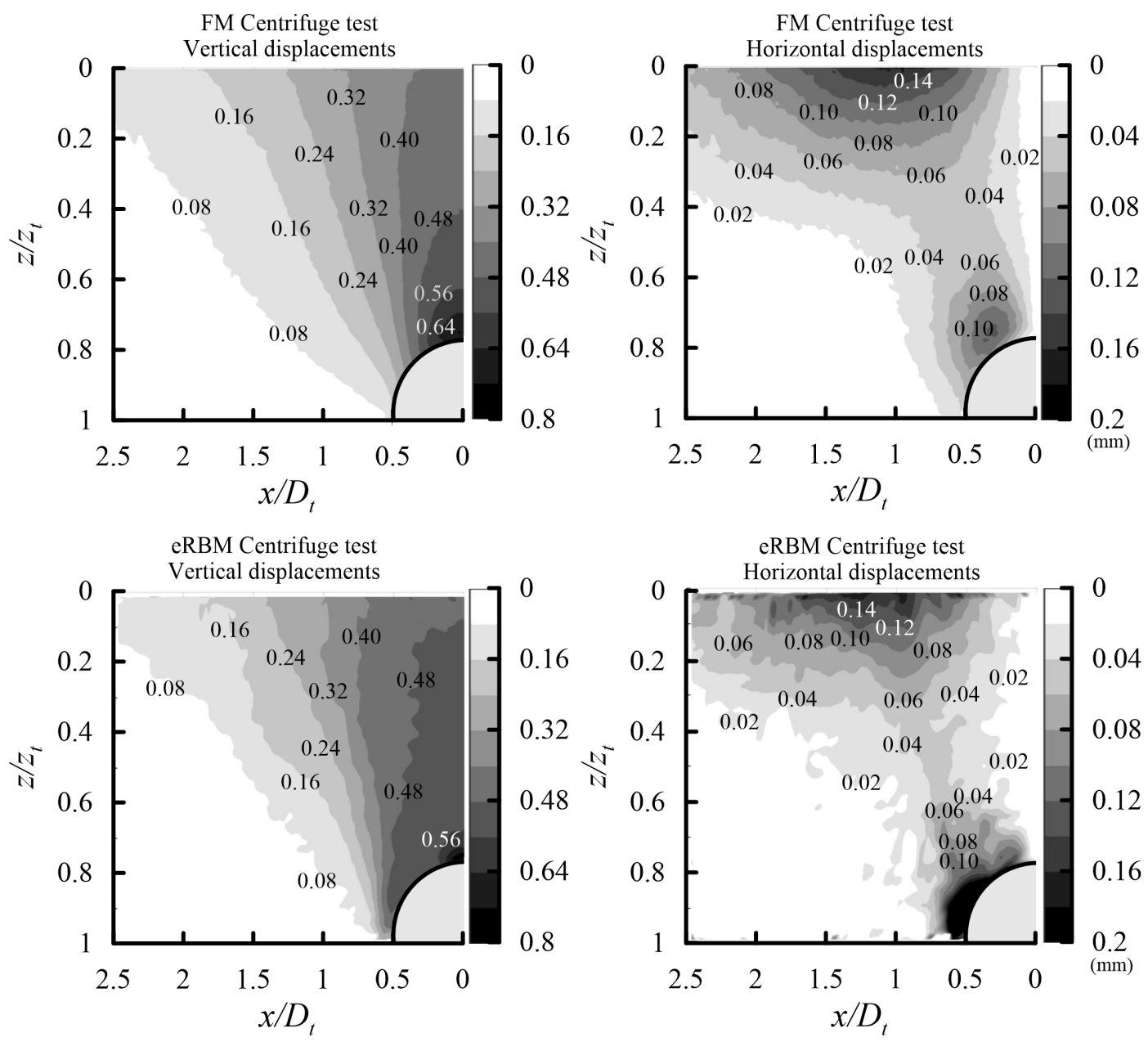

Figure 10: Centrifuge test results of vertical and horizontal soil displacements for FM and eRBM model tunnels: $C / D_{t}=2$ and $V_{l, t}=2 \%$ 
PCM simulation is similar to the FM model tunnel centrifuge test. The magnitude of vertical soil displacements above the tunnel crown in the FEA PCM is larger and more uniformly distributed in the region directly above the tunnel than in the FM centrifuge test. The dominant zone of horizontal displacements for the FEA PCM test is located adjacent to the tunnel springline and creates a larger influence zone than in the FM centrifuge test. The horizontal displacements in the FEA PCM test also show a greater influence zone at the soil surface, with a greater maximum magnitude compared with the FM centrifuge test.

The FEA EDC results in Figure 11 can be directly compared with the eRBM tunnel centrifuge results in Figure 10. The FEA EDC results give larger vertical settlements than the centrifuge test. Similar to the FEA PCM versus FM centrifuge results, the magnitude of vertical displacements for the soil located above the tunnel crown is larger and more uniformly distributed along the depth of the soil in the FEA EDC test compared to the eRBM centrifuge test. The horizontal displacements in the FEA EDC test show a similar pattern to the eRBM centrifuge test, but again displacements are greater and more dispersed in the FEA EDC test. For horizontal displacements, a more concentrated zone of major displacements is shown in the FEA EDC test than in the FEA PCM test.

\subsection{Settlement troughs}

Figure 12 presents the vertical settlement troughs at the surface $\left(z / z_{t}=0\right)$ and subsurface $\left(z / z_{t}=0.5\right)$ for centrifuge tests (refer to Table 1) and numerical (FEA) analyses at a tunnel volume loss of $V_{l, t} \approx 2 \%$. Note that the FM centrifuge test and corresponding FEA PCM analysis for $C / D_{t}=2.4$ have 
FEA PCM

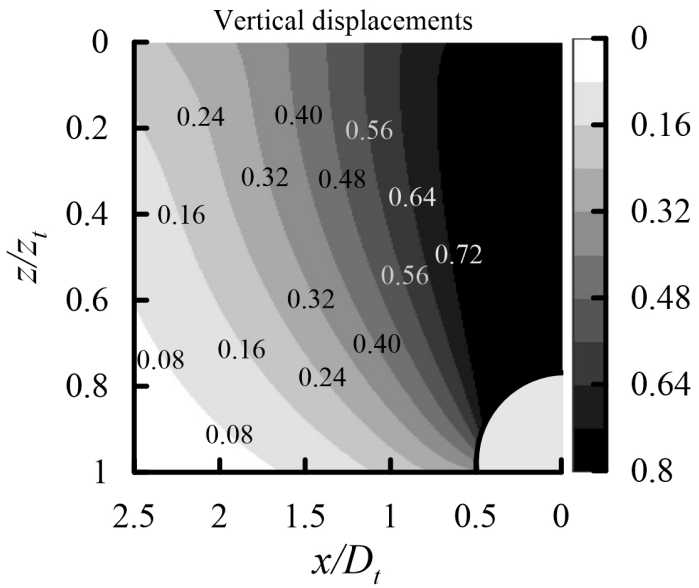

FEA EDC

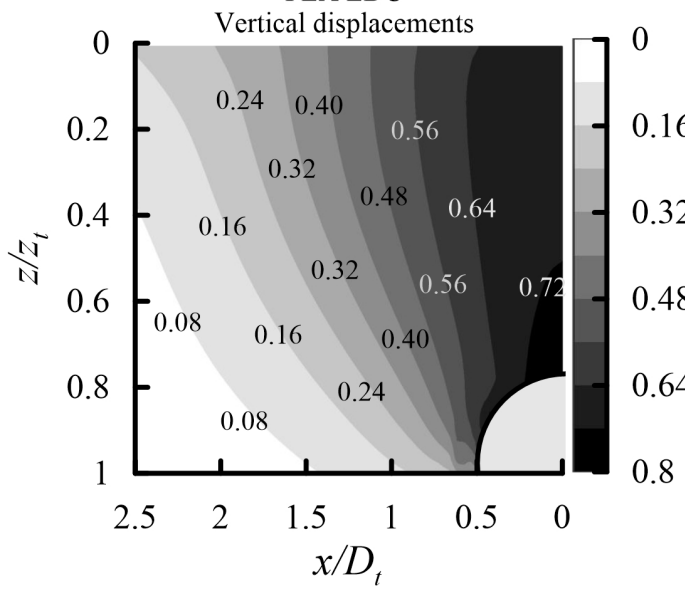

FEA PCM

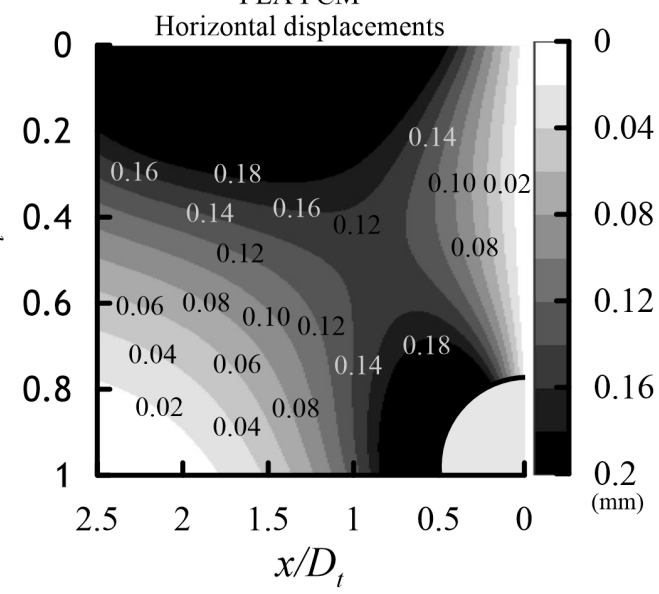

FEA EDC

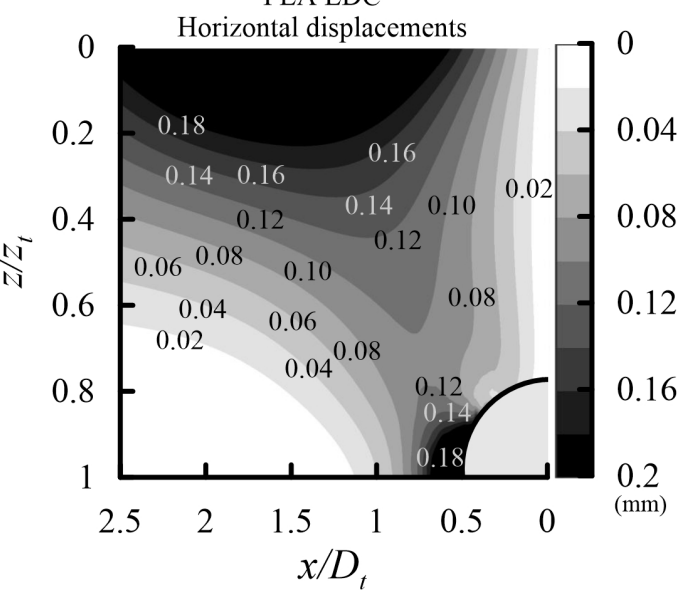

Figure 11: Numerical (FEA) results of vertical and horizontal soil displacements for PCM, EDC, and CDC tunnels: $C / D_{t}=2$ and $V_{l, t}=2 \%\left(\mathrm{PCM} V_{l, t}=1.9 \%\right)$ 
a tunnel diameter $\left(D_{t}=62 \mathrm{~mm}\right)$ that is smaller than other tests (where $D_{t}=90 \mathrm{~mm}$ ); see Table 1. Whilst in dimensionless terms, the $C / D_{t}=2.4$ test should provide a direct comparison to the other tests, this comparison does not consider the fact that, for a given $C / D_{t}$ ratio and tunnel volume loss, a larger tunnel will incur larger magnitude displacements than a smaller tunnel. In addition, for a given $C / D_{t}$, a larger tunnel will be deeper than a smaller tunnel, hence the stress conditions around the larger tunnel will be greater, impacting on the volumetric response of the soil during shear caused by tunnel volume loss (greater stresses resulting in less soil dilation). The impact of this issue will be discussed in relation to the obtained results.

In general, for $C / D_{t}=1.3$ and 2.0 (identical tunnel diameter and similar stress levels between FM and eRBM centrifuge tests), at the given tunnel volume loss of $V_{l, t} \approx 2 \%$, the FM and eRBM centrifuge tests show a similar settlement shape and magnitude at the surface. In addition, the subsurface settlements for both tests are greater than the surface settlement for the soil close to the tunnel centreline; with increase in horizontal distance from the tunnel $x / D_{t}$, subsurface settlement becomes less than at the surface. For $C / D_{t}=2.4$, because the diameter of the FM model tunnel is less than the eRBM model tunnel, for the given value of $V_{l, t}$, the FM model tunnel shows smaller settlements than the eRBM model tunnel. As for $C / D_{t}=1.3$ and 2.0, the shape of the FM and eRBM settlement troughs are similar. The settlement trough shape characteristics are considered later in Figure 18.

For the numerical (FEA) results in Figure 12 (b), as mentioned earlier, the FEA PCM analyses are intended to simulate the FM model tunnel centrifuge tests, and the FEA EDC analyses are intended to replicate the eRBM model 
tunnel centrifuge tests. For $C / D_{t}=1.3$ and 2.0, the FEA PCM settlements are always slightly higher than the corresponding FEA EDC model. As with the centrifuge tests, subsurface settlements close to the tunnel centreline are greater than the surface soil settlement, but becomes less than surface settlement with an increase in horizontal distance from the tunnel $x / D_{t}$. The FEA PCM settlements are generally less than FEA EDC for $C / D_{t}=2.4$, for the same reason previously mentioned for the centrifuge tests (due to the smaller tunnel size in the FEA PCM $C / D_{t}=2.4$ analysis). To compare PCM against EDC numerical results for a consistent tunnel size, an additional FEA PCM analysis was conducted for $C / D_{t}=2.4$ with a tunnel diameter $D_{t}=90 \mathrm{~mm}$ (labelled in Figure $12(\mathrm{~b})$ ). Comparison of the FEA PCM results with $D_{t}=90 \mathrm{~mm}$ against FEA EDC for $C / D_{t}=2.4$ show consistent trends with those previously described for $C / D_{t}=1.3$ and 2.0.

Franza and Marshall (2018) suggested (based on FM centrifuge test data) that a localised zone of large settlements concentrated around the tunnel periphery is indicative of the boundary of an arch; these localised zones can be discerned in Figure 10 for both FM and eRBM centrifuge tests. These localised zones are not observed in the numerical model results in Figure 11, where settlements propagate upwards more gradually.

The numerical (FEA) outcomes in Figure 12 generally over-predict settlements compared to the centrifuge tests. This over-prediction is mainly due to the fact that the numerical analyses were not able to accurately replicate the localised zone of vertical settlement close to the tunnel crown, with tunnel boundary displacements therefore propagating through the soil towards the surface more in the numerical analyses than in the centrifuge tests. As 

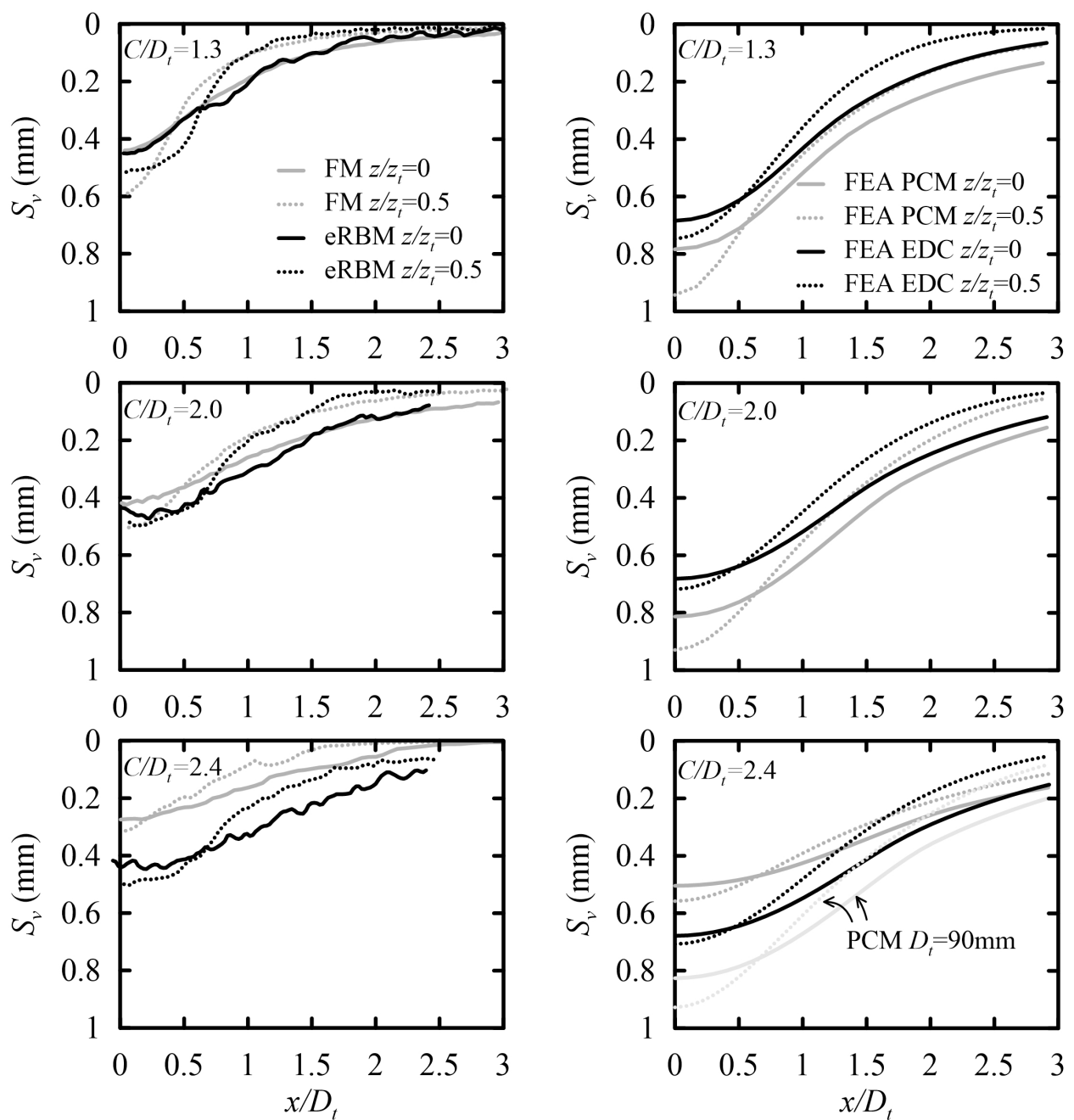

(a)

(b)

Figure 12: Settlement trough data: (a) centrifuge and (b) numerical (FEA) for $V_{l, t} \approx 2 \%$ 
suggested by Franza et al. (2019a), for dense sand, the arching mechanism has an influential impact, and the soil tends to dilate below the developed arch. However, in the numerical simulations, soil dilation was not accurately replicated, which affected the ability of the numerical models to replicate soil displacements.

\subsection{Relationship between soil and tunnel volume loss}

Figure 13 shows the relationship between surface soil volume loss $\left(V_{l, s, s u r f}\right.$; calculated as the integration of surface settlement data) and tunnel volume loss $\left(V_{l, t}\right.$; obtained directly from the displacements or volume changes of the model tunnels) for both eRBM and FM centrifuge tests. Note that for an undrained case (zero volumetric strain), $V_{l, s, s u r f}=V_{l, t}$. As discussed in Marshall et al. (2012) and Franza et al. (2019a), for dry sand (or drained soil conditions), soil volume loss will differ from tunnel volume loss due to the dilative/contractive nature of the soil, which depends on the relative density of the soil, magnitude of the shear strain, and the confining pressures during shear. At a given soil depth, if the value of $V_{l, s, s u r f}$ is lower than $V_{l, t}$, the soil below that level has undergone an overall dilative response.

For the FM model tunnel with $C / D_{t}=1.3$ in Figure $13, V_{l, s, s u r f}$ is always less than $V_{l, t}$, indicating that the soil below the surface experienced an overall dilative response, whereas for $C / D_{t}=2.0$ and 2.4 , the value of $V_{l, s, s u r f}$ is greater than $V_{l, t}$ for tunnel volume losses lower than $V_{l, t} \approx 1.8 \%$ and $2.3 \%$, respectively, indicating an overall contractive response. These trends are consistent with the expected effect of $C / D_{t}$ (for constant tunnel size), where the soil above shallower tunnels is more dilative because of the lower levels of confining stress. The trends of the data for the eRBM model tunnel follow 
those of the FM model tunnel, however the eRBM values of $V_{l, s, s u r f}$ are consistently lower than the FM values, indicating more dilative conditions in the eRBM tests. This suggests that the magnitude of shear strains around the tunnel circumference in the eRBM model tunnel tests were greater than in the FM model tunnel tests. Despite the fact that, because of the different tunnels sizes (see Table 1 ), the $C / D_{t}=2.4$ eRBM tunnel was deeper than the FM tunnel (which would tend to make the eRBM soil more contractive), the eRBM $C / D_{t}=2.4$ results in Figure 13 indicate a more dilative response than the equivalent FM test; this occurs because the larger eRBM tunnel causes higher levels of shear strain than the smaller FM tunnel, resulting in a more dilatant volumetric soil response for the eRBM tunnel (this aspect is discussed in more detail later).

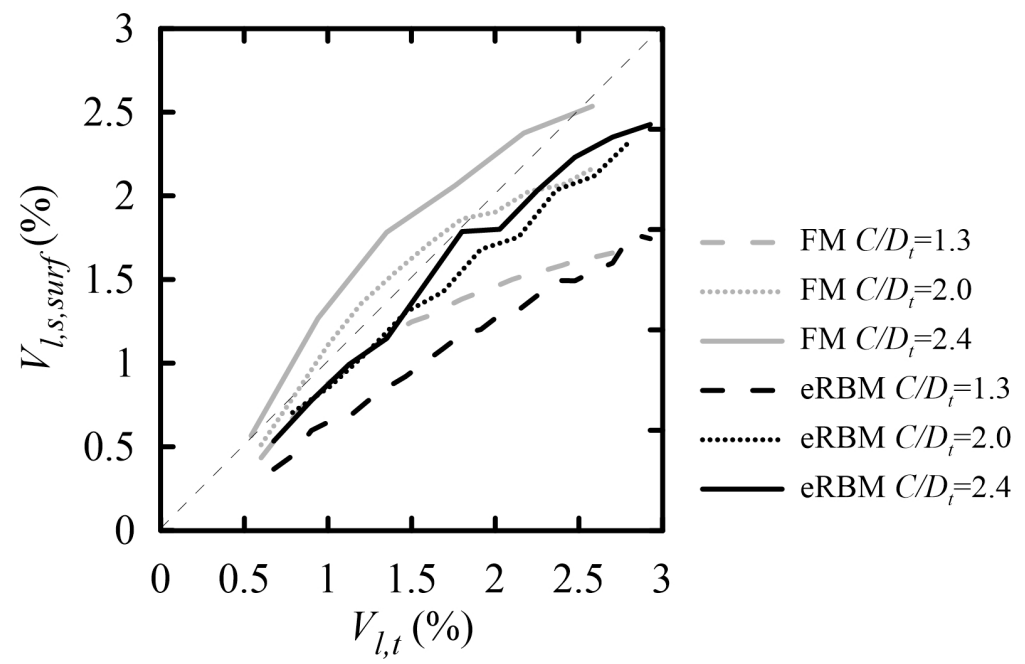

Figure 13: Variation of surface soil volume loss with tunnel volume loss for centrifuge tests

The accumulated soil response indicated by $V_{l, s}$ averages out localised volumetric responses within the soil beneath the calculated level; the soil 
may experience localised contraction at a certain depth yet $V_{l, s}$ could still indicate an overall dilative response. To investigate this feature, Figure 14 plots soil volume loss $\left(V_{l, s}\right)$ with depth at a tunnel volume loss of $V_{l, t} \approx 2.0 \%$ for both centrifuge and numerical tests. The vertical dashed lines indicate a constant-volume condition. For the centrifuge tests, $V_{l, s}$ values are always less than $V_{l, t} \approx 2.0 \%$, apart from the FM $C / D_{t}=2.4$ test, where the cumulative soil response close to the surface $\left(z / z_{t}<0.1\right)$ gives a soil volume loss slightly greater than $2.0 \%$. The value of $V_{l, s}$ for the centrifuge tests reduces approximately linearly with depth $\left(z / z_{t}\right)$ and shows a maximum value at the soil surface and a minimum value close to the tunnel crown. The soil volume loss at a given depth ratio $\left(z / z_{t}\right)$ also differs with $C / D_{t}$ ratio, where a greater $C / D_{t}$ gives a higher soil volume loss (due to higher overall confining stresses, as mentioned earlier). For centrifuge tests with $C / D_{t}=1.3$ (both FM and eRBM), the soil close to the tunnel crown $\left(z / z_{t} \approx 0.7\right)$ shows a value of $V_{l, s}$ that is approximately $50 \%$ of $V_{l, t}$. These data indicate that the soil experienced considerable localised dilation below $z / z_{t} \approx 0.7$. In addition, with a decrease in $z / z_{t}$ (moving away from the tunnel towards the surface), the value of $V_{l, s}$ tends to increase, suggesting that the soil above $z / z_{t}=0.7$ underwent overall contraction.

For greater tunnel depths (greater mean effective stress), the soil will tend to have a more contractive (less dilative) response. The rate of change of contractive response with depth is indicated by the slope of $V_{l, s}$ with depth. The slope for $C / D_{t}=1.3$ for the eRBM centrifuge model tunnel is less than for $C / D_{t}=2.0$ and 2.4, indicating that, for a given shear strain (same tunnel volume loss and tunnel size), the soil in the $C / D_{t}=1.3$ tests is less 
contractive than the soil in the tests with greater tunnel depths $\left(C / D_{t}=2.0\right.$ and 2.4), as is expected.

The centrifuge results show that, for a given value of $C / D_{t}$, the soil volume losses are similar throughout the depth of the soil, with values from the FM tests being marginally larger than the eRBM tests.

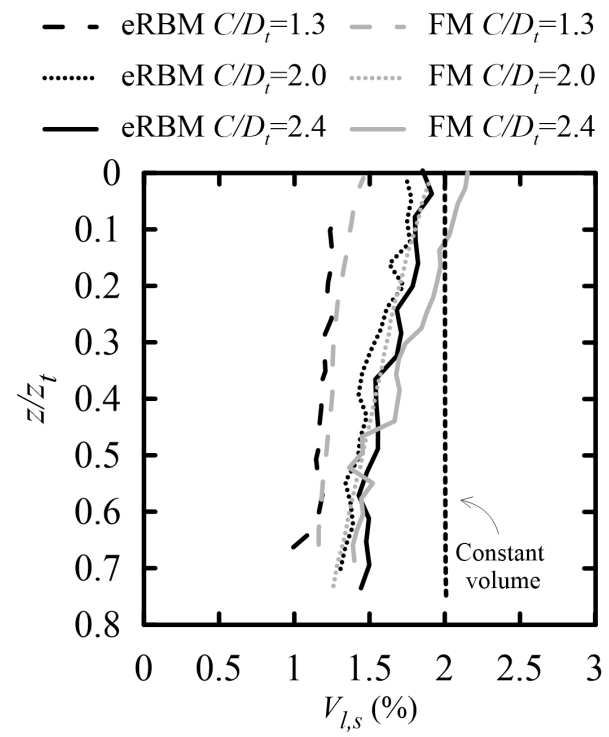

(a)

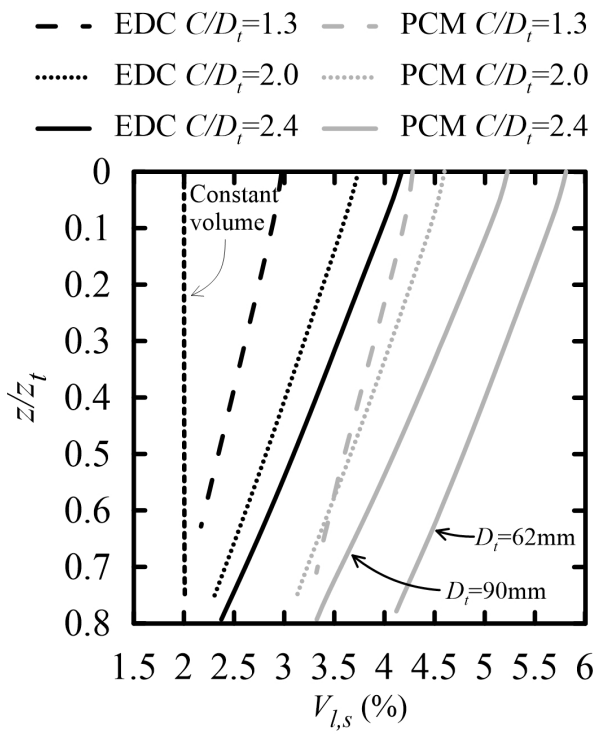

(b)

Figure 14: Variation of $V_{l, s}$ with depth at $V_{l, t} \approx 2.0 \%$ : (a) centrifuge tests; (b) numerical (FEA) simulations

For the numerical (FEA) analyses, both pressure control (PCM) and eccentric displacement control (EDC) methods show soil volume loss is greater than tunnel volume loss across the full range of depth. Consistent with the centrifuge test results, higher $C / D_{t}$ ratios give greater values of $V_{l, s}$. Close to the tunnel crown $(z / z t \approx 0.7)$, unlike the centrifuge tests, the value of $V_{l, s}$ is greater than $2.0 \%$, indicating that the soil below this level underwent contraction, with soil contraction continuing up to the surface. Similar to 
centrifuge test results, but to a much more significant degree, at a given depth $z / z_{t}$, the PCM soil volume losses $V_{l, s}$ are greater than the EDC values (explaining the larger settlements for the PCM results in Figure 12b). The slope of the $V_{l, s}$ data with depth (indicating the rate of change of contractive response with depth) increases with $C / D_{t}$ ratio, similar to the centrifuge test results.

As was done for Figure 12, result from the additional $C / D_{t}=2.4 \mathrm{FEA}$ PCM test with $D_{t}=90 \mathrm{~mm}$ is presented in Figure 14(b). The slope of $V_{l, s}$ with depth is similar for the two PCM analyses for $C / D_{t}=2.4$ in Figure 14(b) $\left(D_{t}=62 \mathrm{~mm}\right.$ from Table 1 and $D_{t}=90 \mathrm{~mm}$ for the additional analysis labelled in the figure). The notable difference between these two analyses is the soil volume loss near the tunnel crown $(z / z t \approx 0.7)$, where $V_{l, s}$ for the analysis with $D_{t}=90 \mathrm{~mm}$ is less than the analysis with $D_{t}=62 \mathrm{~mm}$, indicating that the soil response for $D_{t}=90 \mathrm{~mm}$ was more dilative than for $D_{t}=62 \mathrm{~mm}$. If we consider the mean stresses in the two tests, the analysis with $D_{t}=90 \mathrm{~mm}$ will have a deeper tunnel than for $D_{t}=62 \mathrm{~mm}$ (given the same $C / D_{t}$ ), hence the overall stress conditions will be higher for the $D_{t}=90 \mathrm{~mm}$ analysis, which would imply that the soil should be more contractive, yet this is not what occurred (note that, applying the relative dilatancy concept from Bolton (1986), the relative dilatancy index is reduced from 4.35 for the shallower tunnel to 3.97 for the deeper tunnel, i.e. only a $9 \%$ reduction). The other aspect that can explain the outcome where $V_{l, s}$ for $D_{t}=90 \mathrm{~mm}$ is less than for $D_{t}=62 \mathrm{~mm}$ (i.e. more dilative for the larger tunnel) is the magnitude of shear strain around the tunnels combined with the volumetric response of the soil (illustrated in Figure 6 for triaxial 
conditions). The smaller tunnel will incur lower levels of shear strain than the larger tunnel (this was verified from the FEA analyses), hence the soil near the tunnel for the $D_{t}=62 \mathrm{~mm}$ will be more contractive than the larger tunnel, resulting in the higher levels of soil volume loss at $z / z t \approx 0.7$ in Figure 14(b).

Figure 14 indicates that the numerical analyses did not accurately simulate the soil volumetric behaviour close to the tunnel circumference; below $z / z_{t} \approx 0.7$ (close to the tunnel crown), a significant soil dilative response is observed for the centrifuge test data, whereas a contractive response is obtained in the numerical results. Above $z / z_{t} \approx 0.7$, both centrifuge and numerical results indicate an overall contractive response, though the rate of change of contraction with depth is greater in the FE results than in the centrifuge.

Figure 15 shows the spatial distribution of volumetric and shear strains from the eRBM and FM centrifuge tests for $C / D_{t}=2.0$ at a tunnel volume loss of $V_{l, t}=2.0 \%$ (which have the same size of tunnel; see Table 1). Both model tunnels indicate concentrated zones of shear and negative volumetric (dilation is negative) strain close to the tunnel boundary; near the crown/shoulder for the FM test and closer to the tunnel springline for the eRBM test. The magnitudes of the volumetric strains for the eRBM test are considerably larger than the FM test (the maximum dilative volumetric strain for the eRBM test is approximately nine times higher than the FM test). The magnitudes of shear strain for eRBM test are also significantly higher than the FM test. Theses results confirm the conclusions made earlier (i.e. eRBM values of $V_{l, s, s u r f}$ are consistently lower than the FM values, 
indicating more dilative conditions in the eRBM test). In Figure 15, the highlighted regions of dilating soil are very localised around the tunnel (and below about $\left.z / z_{t}=0.7\right)$; the majority of the soil is contractive, which explains the trends in Figure 14a (i.e. an increase in soil volume loss with a decrease in depth, suggesting contractive soil behaviour above $z / z_{t} \approx 0.7$ ).
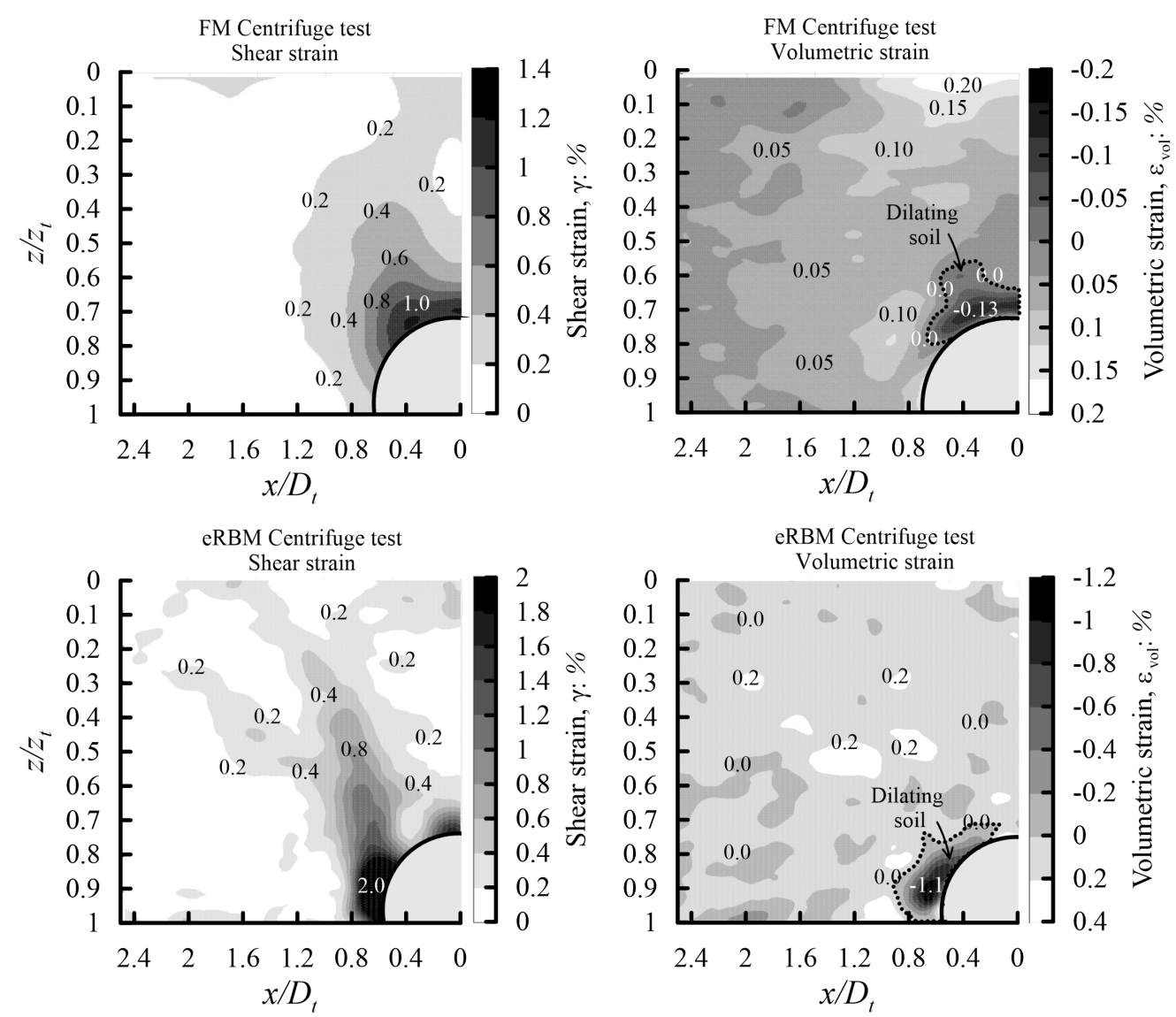

Figure 15: Contours of shear and volumetric strain for centrifuge tests with $C / D_{t}=2.0$ at $V_{l, t}=2.0 \%$

Figure 16 shows the shear and volumetric strain results for the corresponding numerical (FEA) analyses (PCM and EDC). The zone of concen- 


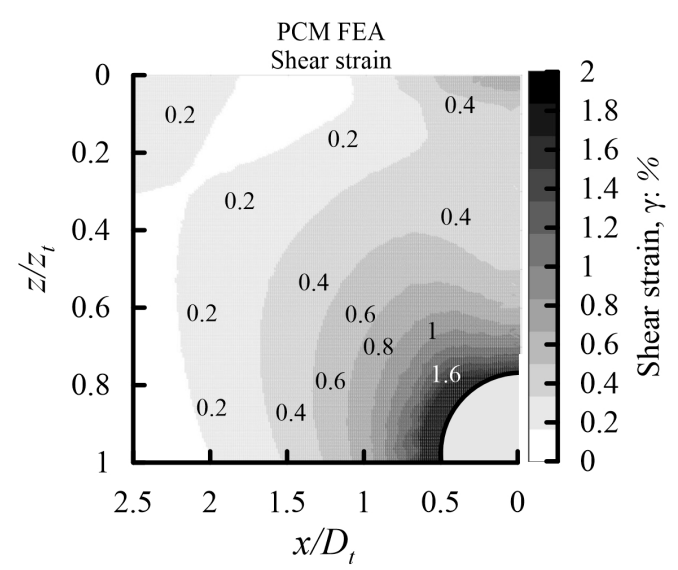

$$
\text { EDC FEA }
$$

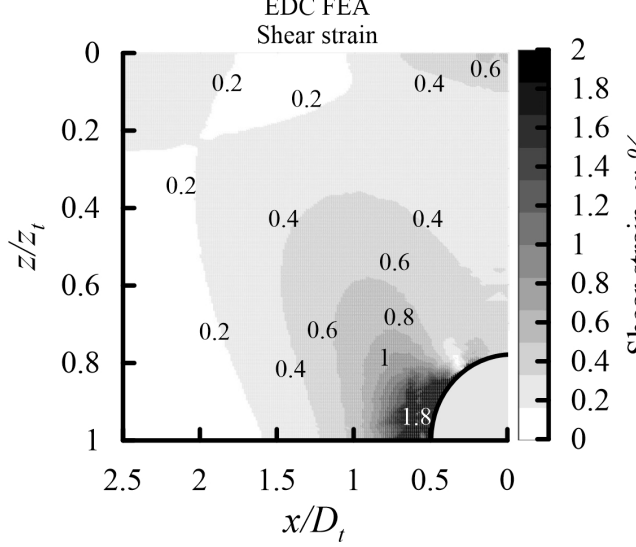
results in the centrifuge tests in Figure 14.

trated shear strain is located at the tunnel shoulders for both methods, with similar magnitude of shear strains as well. There is a region of dilating soil close to the tunnel (highlighted in Figure 16), which is noted to be smaller in size and lower in magnitude than in the centrifuge tests, demonstrating why the numerical model under-predicted the overall dilative soil volume loss
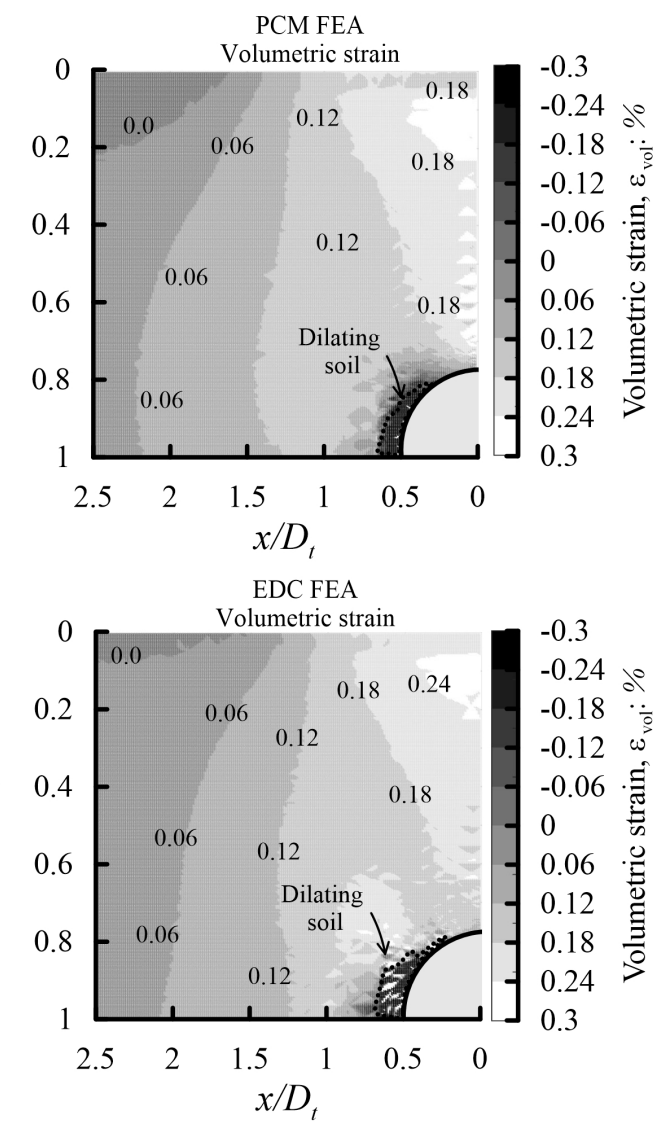

Figure 16: Contours of shear and volumetric strain for numerical (FEA) test with $C / D_{t}=$ 2.0 at $V_{l, t} \approx 2.0 \%$

Figure 17 summarises the key features from the shear and volumetric 
strain distributions of the centrifuge tests. For a given tunnel volume loss, the eRBM model tunnel causes higher magnitude shear strains than the FM model tunnel, resulting in a more dilative volumetric response in the eRBM test than the FM test. The zone of dilating soil for the FM model tunnel is located above the tunnel crown, whereas for the eRBM model tunnel, the zone is located above the tunnel springline. The soil located outside the dilative area undergoes contractive soil behaviour, hence soil volume loss increases as depth decreases.

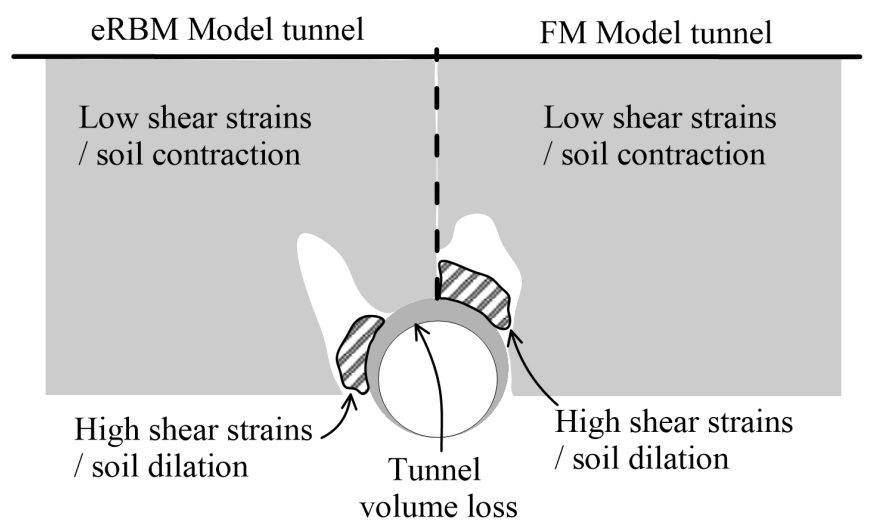

Figure 17: Illustration of soil strains due to tunnel volume loss based on the observations in Figure 15

\subsection{Effect of model tunnel on settlement trough shape characteristics}

To investigate settlement trough shape characteristics, modified Gaussian curves (Vorster et al., 2005) were fitted to the settlement data using the approach of Marshall et al. (2012). Marshall et al. (2012) indicated that the conventional Gaussian curve does not always provide a good fit to settlement data for tunnels in sand, which was substantiated by several other 
studies (Vorster et al., 2005; Marshall et al., 2012; Zhou et al., 2014; Franza and Marshall, 2018; Franza et al., 2019a). The goodness of fit (based on the coefficient of determination $R^{2}$ ) of the modified Gaussian curve to vertical displacements is shown in Figure S1 of the supplemental data. Results demonstrate that the modified Gaussian curve provided a very good fit to the data, with a slight reduction in the goodness of fit to the eRBM data with depth, especially below $z / z_{t}=0.6$. For the numerical analyses, the modified Gaussian curve performed very well for both model types. Analyses presented next are based on the obtained curve fitting results using the modified Gaussian curve.

When applying a modified Gaussian curve, as described by Marshall et al. (2012), the shape of the settlement trough can be described by three points: $S_{\text {max }}, S_{v}\left(i_{g}\right) \approx 0.606 S_{\text {max }}$, and $0.5 S_{v}\left(i_{g}\right) \approx 0.303 S_{\max }$, where $i_{g}$ is the offset from the tunnel centreline to the inflexion point of the standard Gaussian curve. The offset of the second and third points $\left(S_{v}\left(i_{g}\right)\right.$ and $\left.0.5 S_{v}\left(i_{g}\right)\right)$ are referred to as $x^{*}$ and $x^{* *}$, respectively. These offsets can be defined using $x^{*}=K^{*}\left(z_{t}-z\right)$ and $x^{* *}=K^{* *}\left(z_{t}-z\right)$, where $K^{*}$ and $K^{* *}$ are trough width parameters. Figure 18 presents the trough width parameters $K^{*}$ and $K^{* *}$ with soil depth for both centrifuge and numerical (FEA) analyses at a tunnel volume loss of $V_{l, t} \approx 2.0 \%$. Considering first the centrifuge data, the FM model tunnel centrifuge tests give lower trough width parameters than the eRBM model tunnel tests, and the FM $K^{*}$ values do not increase with depth as quickly as they do for the eRBM model tunnel. With an increase in $C / D_{t}$ ratio, the difference in $K^{*}$ and $K^{* *}$ between FM and eRBM model tunnels decreases; at $C / D_{t}=2.4$, the values of $K^{*}$ and $K^{* *}$ are very close for 
$z / z_{t} \leq 0.6$ (despite the fact that the FM and eRBM tunnels for $C / D_{t}=2.4$ differed in size; see Table 1).

Comparing centrifuge data to numerical (FEA) results, Figure 18 shows that the trough width parameters for the centrifuge tests are always lower than the numerical values, and the difference between centrifuge and numerical values is relatively consistent across the range of depth and $C / D_{t}$ ratios. Whilst the numerical results of settlement trough parameters are clearly shifted (increased) compared to the centrifuge data, the trends with depth match relatively well. Results from the additional FEA PCM test for $C / D_{t}=2.4$ with $D_{t}=90 \mathrm{~mm}$ are also presented in Figure 18 (note that the FEA PCM analysis referred to in the legend relates to $D_{t}=62 \mathrm{~mm}$ as it replicated the FM centrifuge test from Table 1). The FEA PCM $D_{t}=90 \mathrm{~mm}$ results match the FEA EDC for $C / D_{t}=2.4$, consistent with $C / D_{t}=2.0$. The numerical results support the conclusion from the centrifuge test data in that, as $C / D_{t}$ increases, the difference between EDC (eRBM) and PCM (FM) decreases. In addition, comparing PCM $D_{t}=90 \mathrm{~mm}$ with PCM $D_{t}=62 \mathrm{~mm}$ shows that, for the given tunnel volume loss, the through width parameters decrease with the increase in tunnel diameter. This outcome is a result of the effect of the higher magnitude shear strains that occur around the larger tunnel on the volumetric soil response, as discussed previously.

Figure 18 demonstrates that, for both FM and eRBM model tunnels, the numerical analyses predict a wider settlement trough than observed experimentally, which can be attributed to the ability of the constitutive model to describe the real stress-strain behaviour of the soil within the centrifuge tests. Figure 16 demonstrated that the volumetric strain behaviour of the 


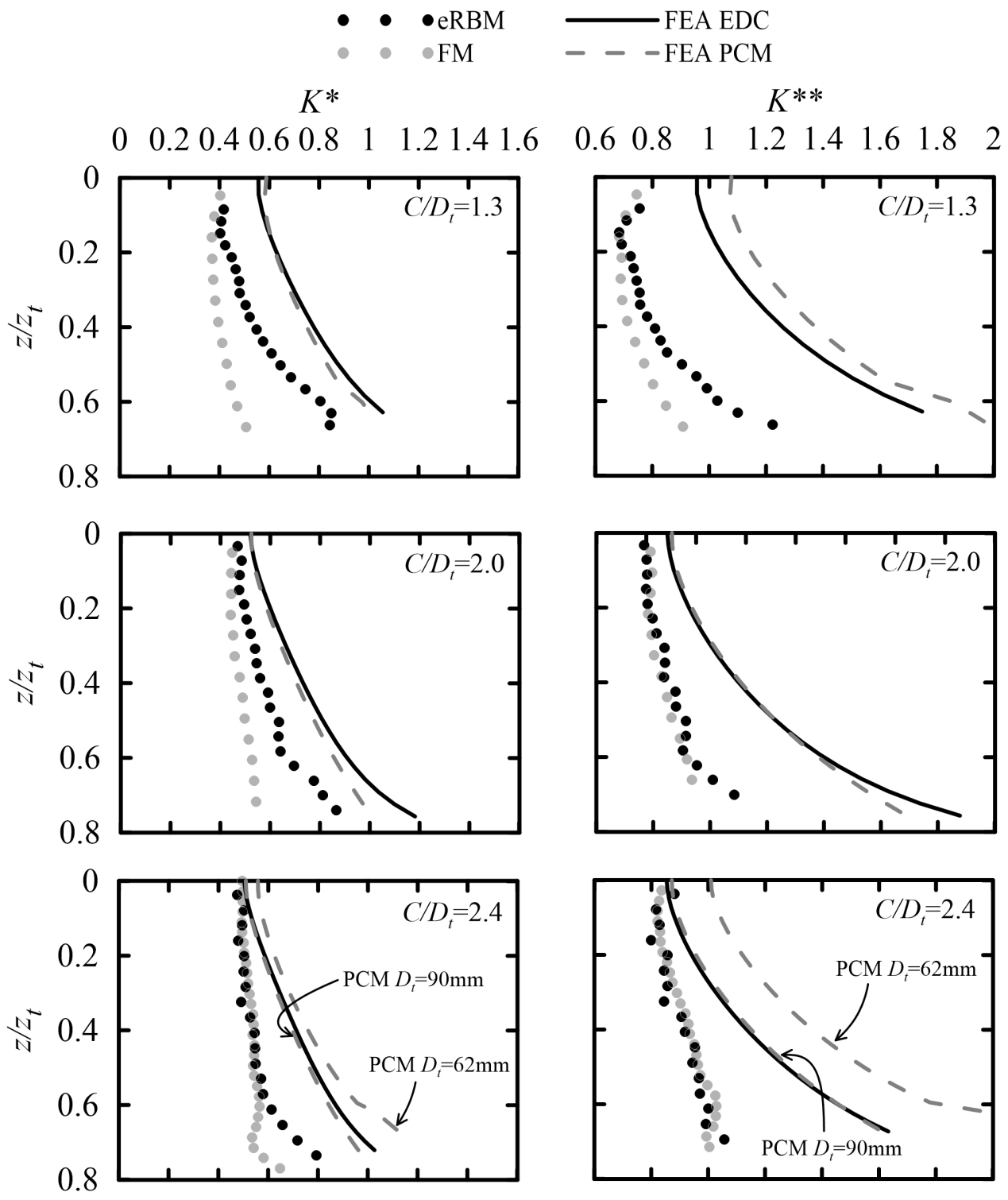

Figure 18: $K^{*}$ and $K^{* *}$ with depth for centrifuge and numerical (FEA) tests at $V_{l, t} \approx 2.0 \%$ 
soil close to the tunnel boundary, in particular the high level of dilation, was not accurately simulated in the numerical analyses, which will affect the settlement trough shape.

The constitutive model adopted within the numerical analyses presented here was the basic hypoplastic model, where the stress path dependent soil stiffness is not considered. The soil elements close to the tunnel boundary will undergo a change in the direction of principal stresses during tunnel volume loss, and the soil elements close to the tunnel springline will experience a stress release process. The stress paths during tunnel volume loss are in a different direction to those during the centrifuge acceleration stage (see Figure 8). Therefore, the soil stiffness predicted by the FE analyses will not be entirely representative of the soil behaviour in the centrifuge tests.

\section{Conclusions}

Two types of model tunnel are commonly adopted to simulate 2D tunnel volume loss in plane strain centrifuge tests: flexible membrane (FM) and rigid boundary mechanical (RBM). This paper provided an in-depth assessment of the effect of the different model tunnel boundary conditions on resulting ground deformations. Finite element analyses were conducted using both pressure and displacement control methods to compare against the centrifuge test data. The paper does not give a recommendation as to which type best replicates reality, as each has advantages and limitations; instead the paper aims to provide readers with quantitative data which will allow them to make an informed decision regarding their choice of model tunnel. The following conclusions can be drawn from the presented results: 
- Centrifuge spin-up has an effect on the initial conditions of the soil during centrifuge tests, which is affected by the type of model tunnel. In a FM model tunnel, the internal tunnel pressure at the tunnel springline is greater than the horizontal soil pressure, and the soil at the springline is pushed outwards during spin-up. For an eRBM model tunnel, the overall shape of the tunnel boundary does not change during spin-up, however some settlement was measured due to the increased self-weight of the tunnel.

- Two numerical analysis approaches were studied: (1) the '2-80 g considered' model which simulated the (nearly) complete centrifuge spin-up process, and (2) a simplified ' $80 \mathrm{~g}$ initially' model, where the initial stress state at $80 \mathrm{~g}$ was imposed. The magnitude of tunnelling related settlements for the '2-80 g considered' models was always slightly greater than the ' $80 \mathrm{~g}$ initially' models for both tunnel types (displacement and pressure controlled).

- Modified Gaussian curves were fitted to the settlement data from the centrifuge and numerical tests. The eRBM model tunnel always provided a wider settlement trough than the FM model tunnel, and settlement troughs from the numerical analyses were considerably wider than the centrifuge test data.

- Centrifuge test data demonstrated that localised zones of high shear strain and soil dilation occur around the model tunnels, though the location of this zone differs between the two model tunnel types. The eRBM generally caused higher levels of shear strain and more dilative 
soil response than the FM model tunnel, which has an impact on the relationship between soil and tunnel volume loss. Numerical analyses were not able to accurately replicate this zone of localised dilation, hence soil volume loss in the numerical analyses was much more contractive than in the centrifuge tests. This issue was a main cause of the discrepancy between centrifuge and numerical results of soil volume loss and settlement trough width. 


\title{
7. NOTATION
}

\author{
$A_{t} \quad$ Tunnel cross-sectional area \\ C Depth of cover above the tunnel \\ $C_{u} \quad$ Coefficient of uniformity \\ $D_{t} \quad$ Diameter of the tunnel \\ $D_{50} \quad$ Average size of the soil particle \\ $e_{c 0} \quad$ Critical void ratio at zero pressure \\ $e_{d 0} \quad$ Minimum void ratio at zero pressure \\ $e_{d} \quad$ Void ratio, depends on $C_{u}$ \\ $e_{i 0} \quad$ Maximum void ratio at zero pressure \\ $e_{\max } \quad$ Maximum void ratio \\ $e_{\min } \quad$ Minimum void ratio \\ $e_{p 0} \quad$ Initial void ratio of compression test at zero pressure \\ $G_{s} \quad$ Specific gravity \\ $h_{s} \quad$ Granular hardness \\ $i$ or $i_{s}$ Horizontal distance between tunnel and inflection point \\ $i_{g} \quad$ Horizontal distance between tunnel and inflection point of greenfield settlements \\ $I_{d} \quad$ Relative density \\ $K \quad$ Trough width parameter \\ $K_{0} \quad$ Static state lateral earth pressure coefficient \\ $K^{*} \quad$ Trough width parameter based on $x^{*}$ \\ $K^{* *} \quad$ Trough width parameter based on $x^{* *}$ \\ $n \quad$ Controls curve fitting parameter \\ $q \quad$ Deviator stress \\ $R^{2} \quad$ Coefficient of determination
}




$\begin{array}{ll}S_{v} & \text { Settlement of soil } \\ S_{\text {max }} & \text { Maximum soil settlement } \\ V_{l, s} & \text { Soil volume loss } \\ V_{l, s, \text { surf }} & \text { Soil volume loss at surface } \\ V_{l, t} & \text { Tunnel volume loss } \\ x & \text { Horizontal distance from the tunnel centre line } \\ x^{*} & \text { Horizontal distance of } S_{v}\left(i_{g}\right) \\ x^{* *} & \text { Horizontal distance of } 0.5 S_{v}\left(i_{g}\right) \\ z_{t} & \text { Depth of the tunnel axis } \\ z & \text { Depth measure from soil surface } \\ \alpha & \text { Governs the peak friction angle of the soil } \\ \beta & \text { Governs the soil stiffness } \\ \gamma & \text { Shear strain } \\ \epsilon_{v o l} & \text { Volumetric strain } \\ \phi_{c}^{\prime} & \text { Critical state friction angle } \\ \sigma_{v} & \text { Vertical stress } \\ \sigma_{v, t} & \text { Vertical stress at tunnel depth } \\ \sigma_{h} & \text { Horizontal stress }\end{array}$




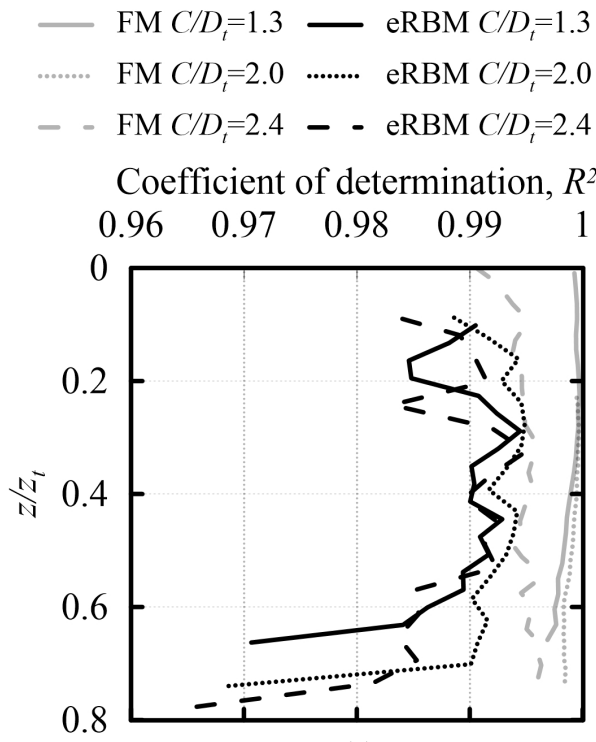

(a)

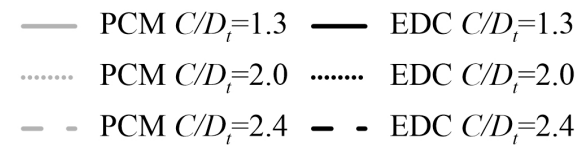

Coefficient of determination, $R^{2}$

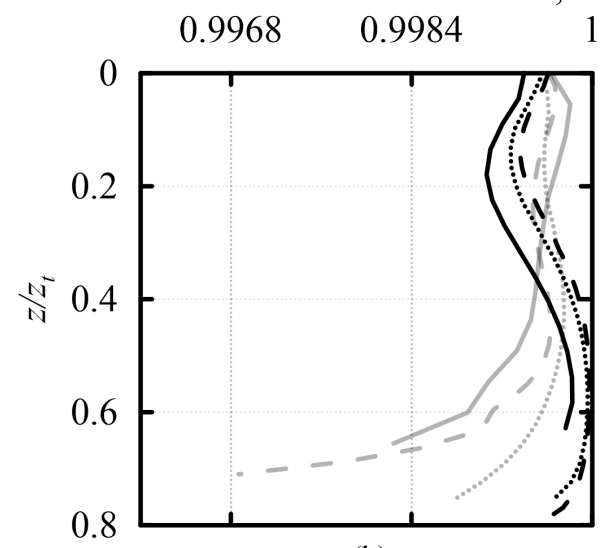

(b)

Figure S1: Goodness of fit of modified Gaussian curves to (a) centrifuge, and (b) numerical (FEA) data 


\section{References}

Atkinson, J. H., Brown, E. T., Potts, D. M., 1975. Collapse of shallow unlined tunnels in dense sand. Tunnels and Tunneling 7 (3), 81-87.

Bolton, M. D., 1986. The strength and dilatancy of sands. Geotechnique $36(1), 65-78$.

Boonsiri, I., Takemura, J., 2015a. A centrifuge model study on pile group response to adjacent tunnelling in sand. Journal of JSCE 3 (1), 1-18.

Boonsiri, I., Takemura, J., 2015b. Observation of ground movement with existing pile groups due to tunneling in sand using centrifuge modelling. Geotechnical and Geological Engineering 33 (3), 621-640.

Cheng, C. Y., Dasari, G. R., Chow, Y. K., Leung, C. F., 2007. Finite element analysis of tunnel-soil-pile interaction using displacement controlled model. Tunnelling and Underground Space Technology 22 (4), 450-466.

Elshafie, M. Z. E. B., Choy, C. K. C., Mair, R. J., 2013. Centrifuge modeling of deep excavations and their interaction with adjacent buildings.

Farrell, R. P., 2010. Tunnelling in sands and the response of buildings. Ph.D. thesis, University of Cambridge.

Franza, A., 2016. Tunnelling and its effects on piles and piled structures. Ph.D. thesis, University of Nottingham.

Franza, A., Marshall, A. M., 2018. Centrifuge modelling study of the response of piled structures to tunnelling. ASCE Journal of Geotechnical and Geoenvironmental Engineering 144 (2), 04017109. 
Franza, A., Marshall, A. M., Zhou, B., 2019a. Greenfield tunnelling in sands: the effects of soil density and relative depth. Geotechnique 69 (4), 297-307.

Franza, A., Marshall, A. M., Zhou, B., Shirlaw, N., Boone, S., 2019b. Discussion: Greenfield tunnelling in sands: the effects of soil density and relative depth. Géotechnique (DOI:10.1680/jgeot.19.d.002).

Gue, C. Y., Elshafie, M. Z. E. B., 2019. Development of a staged volume loss tunnelling method for three-dimensional tunnelling applications in the geotechnical centrifuge. International Journal of Physical Modelling in Geotechnics (DOI:10.1680/jphmg.17.00057).

Hagiwara, T., Grant, R. J., Calvello, M., Taylor, R. N., 1999. The effect of overlying strata on the distribution of ground movements induced by tunnelling in clay. Soils and Foundations 39 (3), 63-73.

Haji, T. K., Marshall, A. M., Franza, A., 2018. Mixed empirical-numerical method for investigating tunnelling effects on structures. Tunnelling and Underground Space Technology 73 (March), 92-104.

Herle, I., Gudehus, G., 1999. Determination of parameters of a hypoplastic constitutive model from properties of grain assemblies. Mechanics of Cohesive-frictional Materials 4 (5), 461-486.

Hibbitt, K., 2002. ABAQUS/Explicit User's Manual: Version 6.3. Hibbit, Karlsonn \& Sorensen.

Jacobsz, S. W., 2003. The effects of tunnelling on piled foundations. Ph.D. thesis, University of Cambridge. 
Katoh, Y., Miyake, M., Wada, M., 1998. Ground deformation around shield tunnel. In: Centrifuge. Vol. 98. pp. 733-738.

Loganathan, N., Poulos, H. G., 1998. Analytical prediction for tunnelinginduced ground movements in clays. Journal of Geotechnical and Geoenvironmental Engineering.

Loganathan, N., Poulos, H. G., Stewart, D. P., 2000. Centrifuge model testing of tunnelling-induced ground and pile deformations. Geotechnique 50 (3), $283-294$.

Marshall, A. M., 2009. Tunnelling in sand and its effect on pipelines and piles. Ph.D. thesis, University of Cambridge.

Marshall, A. M., Elkayam, I., Klar, A., Mair, R. J., 2010. Centrifuge and discrete element modelling of tunnelling effects on pipelines. In: Springman, S. M., Laue, J., Seward, L. (Eds.), 7th International Conference on Physical Modelling in Geotechnics. Vol. v1. Taylor and Francis Group, London, pp. 633-637.

Marshall, A. M., Farrell, R. P., Klar, A., Mair, R. J., 2012. Tunnels in sands: the effect of size, depth and volume loss on greenfield displacements. Géotechnique 62 (5), 385-399.

Marshall, A. M., Franza, A., 2017. Discussion of "Observation of Ground Movement with Existing Pile Groups Due to Tunneling in Sand Using Centrifuge Modelling" by Ittichai Boonsiri and Jiro Takemura. Geotechnical and Geological Engineering 35 (1), 535-539. 
Marshall, A. M., Mair, R. J., 2011. Tunneling beneath driven or jacked endbearing piles in sand. Canadian Geotechnical Journal 48 (12), 1757-1771.

Ng, C. W. W., Lu, H., Peng, S. Y., 2013. Three-dimensional centrifuge modelling of the effects of twin tunnelling on an existing pile. Tunnelling and Underground Space Technology 35, 189-199.

Nomoto, T., Imamura, S., Hagiwara, T., Kusakabe, O., Fujii, N., 1999. Shield tunnel construction in centrifuge. Journal of geotechnical and geoenvironmental engineering 125 (4), 289-300.

Potts, D. M., 1976. Behaviour of lined and unlined tunnels in sand. Ph.D. thesis, University of Cambridge.

Potts, D. M., Zdravković, L., 1999. Finite element analysis in geotechnical engineering: Theory. Vol. 1. Thomas Telford.

Ritter, S., Giardina, G., DeJong, M. J., Mair, R. J., 2017. Centrifuge modelling of building response to tunnel excavation. International Journal of Physical Modelling in Geotechnics, 1-16.

Shahin, H. M., Nakai, T., Zhang, F., Kikumoto, M., Nakahara, E., 2011. Behavior of ground and response of existing foundation due to tunneling. Soils and Foundations 51 (3), 395-409.

Song, G., Marshall, A. M., Heron, C., 2018. A mechanical displacement control model tunnel for simulating eccentric ground loss in the centrifuge. In: 9th International Conference of Physical Modelling in Geotechnics: ICPMG. 
Stanier, S. A., Blaber, J., Take, W. A., White, D., 2015. Improved imagebased deformation measurement for geotechnical applications. Canadian Geotechnical Journal 53 (5), 727-739.

Tan, F. S. C., 1990. Centrifuge and theoretical modelling of conical footings on sand. Ph. D thesis, Cambridge University.

von Wolffersdorff, P.-A., 1996. A hypoplastic relation for granular materials with a predefined limit state surface. Mechanics of Cohesive-frictional Materials 1 (3), 251-271.

Vorster, T. E. B., 2006. The effects of tunnelling on buried pipes. Ph.D. thesis, University of Cambridge.

Vorster, T. E. B., Klar, A., Soga, K., Mair, R. J., 2005. Estimating the effects of tunneling on existing pipelines. Journal of Geotechnical and Geoenvironmental Engineering 131 (11), 1399-1410.

Williamson, M., 2014. Tunnelling effect on bored piles in clay. Ph.D. thesis, University of Cambridge.

Xu, J., Marshall, A. M., Franza, A., Boldini, D., Amorosi, A., DeJong, M. J., 2019. The response of framed buildings on raft foundations to tunnelling: a centrifuge and numerical modelling study. In: Proceedings of the 17th European Conference on Soil Mechanics and Geotechnical Engineering, Reykjavik, Iceland. DOI:10.32075/17ECSMGE-2019-0134.

Youd, T. L., 1973. Factors controlling maximum and minimum densities of sands. In: Evaluation of relative density and its role in geotechnical projects involving cohesionless soils. ASTM International. 
${ }_{952}$ Zhou, B., 2015. Tunnelling-induced ground displacements in sand. Ph.D. ${ }_{953}$ thesis, University of Nottingham.

${ }_{954}$ Zhou, B., Marshall, A. M., Yu, H. S., 2014. The effect of relative density 955 on greenfield settlements above tunnels in sands. In: Geoshanghai 2014 956 - International conference on geotechnical engineering. ASCE, Shanghai, 957 pp. $96-105$. 